\title{
One-dimensional bargaining: a revision
}

Citation for published version (APA):

Predtetchinski, A. (2010). One-dimensional bargaining: a revision. METEOR, Maastricht University School of Business and Economics. METEOR Research Memorandum No. 031

https://doi.org/10.26481/umamet.2010031

Document status and date:

Published: 01/01/2010

DOI:

10.26481/umamet.2010031

Document Version:

Publisher's PDF, also known as Version of record

\section{Please check the document version of this publication:}

- A submitted manuscript is the version of the article upon submission and before peer-review. There can be important differences between the submitted version and the official published version of record.

People interested in the research are advised to contact the author for the final version of the publication, or visit the DOI to the publisher's website.

- The final author version and the galley proof are versions of the publication after peer review.

- The final published version features the final layout of the paper including the volume, issue and page numbers.

Link to publication

\footnotetext{
General rights rights.

- You may freely distribute the URL identifying the publication in the public portal. please follow below link for the End User Agreement:

www.umlib.nl/taverne-license

Take down policy

If you believe that this document breaches copyright please contact us at:

repository@maastrichtuniversity.nl

providing details and we will investigate your claim.
}

Copyright and moral rights for the publications made accessible in the public portal are retained by the authors and/or other copyright owners and it is a condition of accessing publications that users recognise and abide by the legal requirements associated with these

- Users may download and print one copy of any publication from the public portal for the purpose of private study or research.

- You may not further distribute the material or use it for any profit-making activity or commercial gain

If the publication is distributed under the terms of Article $25 \mathrm{fa}$ of the Dutch Copyright Act, indicated by the "Taverne" license above, 


\section{Maastricht University}

Arkadi Predtetchinski

One-dimensional bargaining: a revision

$\mathrm{RM} / 10 / 031$

\section{METEOR}

Maastricht University School of Business and Economics

Maastricht Research School of Economics

of Technology and Organization

P.O. Box 616

NL - 6200 MD Maastricht

The Netherlands 


\title{
One-dimensional bargaining: a revision
}

\author{
Arkadi Predtetchinski ${ }^{1}$
}

July 1, 2010

\begin{abstract}
We study a model of multilateral bargaining over social outcomes represented by the points in the unit interval. The acceptance or rejection of a proposal is determined by an acceptance rule represented by the collection of decisive coalitions. The focus of the paper is on the asymptotic behavior of subgame perfect equilibria in stationary strategies as the players become infinitely patient. We show that, along any sequence of stationary subgame perfect equilibria the social acceptance set collapses to a point. This point, called the limit of bargaining equilibria, is independent of the sequence of equilibria and is uniquely determined by the set of players, the utility functions, the recognition probabilities, and the acceptance rule. The central result of the paper is a characterization of the limit of bargaining equilibria as a unique zero of the characteristic equation. The characterization is used to study the comparative statics of the limit of bargaining equilibria with respect to the acceptance rule.
\end{abstract}

JEL classification code: C78

Keywords: Bargaining, subgame perfect equilibrium, acceptance rules.

\footnotetext{
${ }^{1}$ Department of Economics, Maastricht University, P.O. Box 616, 6200 MD Maastricht, The Netherlands. E-mail: A.Predtetchinski@maastrichtuniversity.nl. The author acknowledges the financial support of the Dutch Science Foundation (NWO) through a VENI-grant. The author is most thankful to Jean-Jacques Herings and the anonymous referees for their suggestions.
} 


\section{Introduction}

This paper analyzes a model of multilateral bargaining where players must choose one alternative from a set of alternatives represented by points in the unit interval. An alternative might be a level of taxation, a location of a facility, or an index of an ideological content of a policy (left vs. right).

Bargaining proceeds as follows. At the beginning of each period, nature randomly selects one of the players as a proposer. The probability for a player to become a proposer, the so-called recognition probability, is assumed to be the same in each period. The player chosen by nature puts forward a proposal specifying one alternative. All players then react to the proposal. Each player can either reject or accept the proposal. Whether the proposal passes or fails is then determined by the acceptance rule, as represented by the collection of decisive coalitions. The passing of a proposal requires an approval of it by all the players in some decisive coalition. Examples of acceptance rules are the unanimity acceptance rule when a passing of a proposal requires an approval of it by all the players, and the quota rule, when a fixed number of votes is needed for a passing of a proposal. If the proposal passes, it is implemented and the game ends. In this case each player receives a discounted utility of the implemented alternative. Otherwise, a new period begins.

We consider subgame perfect equilibria in stationary strategies. Stationarity means that a proposal of any player does not depend on the history of play and a player's reaction to a proposal depends only on the proposal itself. The focus of the paper is on the asymptotic behavior of stationary subgame perfect equilibria as the discount factor approaches one.

We prove that subgame perfect equilibria in stationary strategies are asymptotically unique in the following sense: Along any sequence of subgame perfect equilibria in stationary strategies the social acceptance set collapses to a point. This point, called the limit of bargaining equilibria, is independent of the sequence of equilibria and is uniquely determined by the set of players, the utility functions, the recognition probabilities, and the acceptance rule. The central result of the paper is a characterization of the the limit of bargaining equilibria as a unique zero of the characteristic equation.

The results are obtained under rather minimal assumptions. Thus the instantaneous utility functions are only assumed to be single-peaked and concave. Furthermore, we require that the intersection of any two decisive coalitions contain a player with a positive recognition probability. This requirement puts but a very mild restriction on the acceptance rule and the recognition probabilities.

Various results on one-dimensional bargaining have been previously obtained in Banks and Duggan [1], Cho and Duggan [5], Kalandrakis [12], Cardona and Ponsati [4], Herings and Predtetchinski [11], Imai and Salonen [9], Compte and Jehiel [6]. Banks and Duggan [1] consider bargaining in a situation where the alternatives are represented by points in a general compact convex set. For the special case where the set of alternatives is onedimensional they establish the existence of stationary subgame perfect equilibria in pure strategies.

The question of uniqueness of stationary subgame perfect equilibria in the one-dimensional bargaining game is addressed in Cho and Duggan [5], Cardona and Ponsati [4], Herings and 
Predtetchinski [11]. Cho and Duggan [5] derive the uniqueness of subgame perfect equilibria in pure stationary strategies assuming that the utility functions are quadratic and the acceptance rule is proper and strong. Cardona and Ponsati [4] show that stationary subgame perfect equilibria in pure strategies are unique in a game where the proposers rotate in a fixed sequence, provided that each player's utility function is symmetric around the peak and the acceptance rule is a quota rule. Herings and Predtetchinski [11] establish the uniqueness result in a model where the identity of the proposer follows a general Markov process, assuming tent-shaped utility functions and the unanimity acceptance rule.

In general, a one-dimensional game of bargaining can have many stationary subgame perfect equilibria, examples of multiplicity given in Cho and Duggan [5] and Kalandrakis [12]. In particular, the 7-player game in Kalandrakis [12] has a continuum of pure strategy stationary subgame perfect equilibria. As is demonstrated in Cho and Duggan [5], stationary equilibria are nested in the sense that the social acceptance set in one equilibrium forms the subset of the social acceptance set of the other equilibrium. Moreover, Kalandrakis [12] shows that pure strategy stationary subgame perfect equilibria are locally unique and finite in number for almost all discount factors.

Asymptotic uniqueness of stationary subgame perfect equilibria in the one-dimensional bargaining game has been shown in Cardona and Ponsati [4]. In this paper not only do we show that equilibria are asymptotically unique, but we also provide a description of the limit by means of a characteristic equation. A characterization of the limit of equilibria is also given in Herings and Predtetchinski [11], but this result applies only to a game with the unanimity acceptance rule and the tent-shaped utility functions. In the case of time-invariant recognition probabilities the characterization of Herings and Predtetchinski [11] follows as a corollary from our main result.

Imai and Salonen [9] introduce the concept of representative Nash bargaining solution for the situation of two-sided bargaining. In a two-sided bargaining problem the alternatives are represented by points in the interval and the players are divided into two groups with diametrically opposite preferences. The authors provide an axiomatization of the representative Nash bargaining solution and the non-cooperative characterization of it as a limit of stationary equilibrium in a game of bargaining when the probability of the breakdown of negotiations vanishes. Unlike Imai and Salonen (2000), we allow for players to have intermediate preferences.

Compte and Jehiel [6] study the performance of majority rules in bargaining assuming that the proposals at any round of negotiations are drawn by nature randomly from a fixed distribution over the set of alternatives, so the players have no influence on the proposals. They demonstrate that the set of accepted proposals shrinks to a point as the players become infinitely patient, and provide a characterization of the limit proposal. When the set of alternatives is one-dimensional they show that the limit proposal is determined by the bliss points of only two players (called by the authors the "decisive" players). For the bargaining with unanimous consent, the decisive players are the players with the lowest and the highest ideal points. Furthermore, the limit proposal coincides with the Nash bargaining solution of the game where only the two decisive players are present. Both Compte and Jehiel [6] and Banks and Duggan [1] also find that under the simple majority 
rule the limit of stationary equilibria coincides with the ideal point of the median player.

Numerous contributions study stationary subgame perfect equilibria in games of multilateral bargaining where the players have to agree upon a division of an amount of money among themselves, in which case the alternatives are represented by points in the simplex. Thus Merlo and Wilson [15, 16] give sufficient conditions for the uniqueness of stationary subgame perfect equilibria (in pure strategies) in a model where the identity of the proposer and the amount of money to be divided follow a Markov process and the unanimous approval is needed for a proposal to pass. Eraslan [7] establishes uniqueness of stationary subgame perfect equilibria (in mixed strategies) in a game with a tree similar to the one described above and the quota acceptance rule. Eraslan and Merlo [8] characterize stationary subgame perfect equilibria (in mixed strategies) in a model where the amount of money to be divided is stochastic and a general agreement rule is used.

The study of the asymptotic behavior of stationary subgame perfect equilibria in Hart and Mas-Colell [10], Miyakawa [17], Laruelle and Valenciano [14], and Britz et al [3] provides a non-cooperative foundation for the asymmetric Nash bargaining solution. It is shown that the limit of stationary subgame perfect equilibria in the $n$-player game as the probability of the breakdown of negotiations goes to zero converges to the asymmetric Nash bargaining solution, with the weights given by players' recognition probabilities. Compte and Jehiel [6] obtain a similar result in a model where the proposals are randomly drawn by nature rather than being chosen by the players. Kultti and Vartiainen [13] obtain the asymmetric Nash bargaining solution as a limit of the Von Neumann-Morgenstern stable set as the discount factor vanishes. The stable set is defined with respect to a dominance relation where an alternative $x$ dominates an alternative $y$ if some player prefers $x$ even with a one-period delay.

In contrast, we show that the limit of stationary subgame perfect equilibria in the onedimensional bargaining model is generally not equal to the asymmetric Nash bargaining solution. The reason for this apparent discrepancy in the results is that the papers that give a non-cooperative foundation for the asymmetric Nash bargaining solution do so under the assumption that the set of feasible utilities has a smooth and non-level boundary. This assumption is typically satisfied in the divide-the-dollar game, for instance, but it is incompatible with the model where the set of alternatives is one-dimensional unless there are only two players.

The rest of the paper is as follows. Section 2 introduces the game of bargaining and the concept of bargaining equilibrium. In Section 3 we establish that, along any sequence of bargaining equilibria, as the discount factor converges to one, the social acceptance set collapses to a point. Any such point is called a limit of bargaining equilibria. Section 4 presents the main results of the paper: the uniqueness of the limit of bargaining equilibria and its characterization as a zero of the characteristic function. The proof of the main results can be found in the Appendix.

In Section 5 we study the comparative statics of the limit of bargaining equilibria with respect to the acceptance rule. In Section 6 we discuss to extensions of the model: we show how to explicitly introduce the disagreement payoffs and how to extend the results of the paper to a setup with asymmetric discount factors. 


\section{Bargaining equilibria}

The unit interval $[0,1]$ represents the set of alternatives or social states the players must choose from. Let $N$ be a finite set of players and let $\mu$ be a probability distribution on $N$. The probability $\mu(i)$ is a recognition probability of player $i$, that is the probability for player $i$ to become a proposer. The utility of the alternative $x \in[0,1]$ to player $i \in N$ is denoted by $u_{i}(x)$. We shall assume that the utility functions satisfy the following assumption.

(A1) For each $i \in N$ the utility function $u_{i}:[0,1] \rightarrow[0,1]$ is concave, continuous, and it attains its unique maximum at point $\bar{x}_{i}$.

The symbol $\mathscr{C}$ denotes a collection of subsets of $N$ representing an acceptance rule. The sets in $\mathscr{C}$ are decisive coalitions in the sense that an approval of a proposal by any of these coalitions is sufficient for a passing of a proposal. For instance, if the passing of a proposal requires the approval of it by each player, then the collection $\mathscr{C}$ consists of the set $N$ alone. If the acceptance of a proposal requires the approval of it by at least $q$ voters, then the the collection $\mathscr{C}$ consists of all sets $C \subset N$ such that $|C| \geq q$. An interesting acceptance rule is an adaptation of the rule used by the United Nations Security Council. The Council consists of five permanent and ten temporary members, and the passing of its resolutions requires the yes-votes of at least nine members. Furthermore, each permanent member has a veto right, which means that the no-vote by any of the permanent members overrules any majority. Letting $P$ denote the set of permanent members, the collection $\mathscr{C}$ consists of all sets $C \subset N$ such that $P \subset C$ and $|C| \geq 9$. We shall assume the following.

(A2) The collection $\mathscr{C}$ is non-empty and monotone: If $C \subset T \subset N$ and $C \in \mathscr{C}$, then $T \in \mathscr{C}$. Furthermore, for each $C_{1}$ and $C_{2}$ in $\mathscr{C}$ the inequality $\mu\left(C_{1} \cap C_{2}\right)>0$ holds.

The condition that any pair of decisive coalitions have a non-empty intersection is known as properness. Properness is clearly a necessary condition for (A2) which requires that the intersection of any pair of decisive coalitions contain a player with a positive probability of recognition. Assumption (A2) is trivially satisfied in the case of the unanimity acceptance rule, i.e. when $\mathscr{C}=\{N\}$. If the number of players is odd and the collection $\mathscr{C}$ represents the simple majority rule, then assumption (A2) implies that all players' recognition probabilities are positive, because for each $i \in N$ there exist two decisive coalitions $C_{1}$ and $C_{2}$ such that $C_{1} \cap C_{2}=\{i\}$. If $\mathscr{C}$ represents the acceptance rule of the Security Council as described above, for the assumption (A2) to be satisfied it is sufficient that at least one permanent member has a positive recognition probability.

Let $\delta$ denote the discount factor. Throughout the paper we assume that $0 \leq \delta<1$. Given $\delta$, consider the game $\Gamma(\delta)$ defined below. Notice that the game tree of $\Gamma(\delta)$ is exactly the same as in Cho and Duggan [5] and Banks and Duggan [1].

The game starts in period zero. Each period $t$ begins with nature randomly choosing a player from the set $N$ to make a proposal. Player $i$ is chosen by nature with the probability of $\mu(i)$. The chosen player proposes an alternative $x$ from $[0,1]$. All players (including the proposer) respond simultaneously to the proposal. Each responder can either accept or 
reject the current proposal. If all players in some decisive coalition $C \in \mathscr{C}$ accept the proposal, the game terminates and the proposal is implemented. Otherwise, period $t+1$ begins.

If alternative $x$ is agreed upon in period $t$, player $i$ receives a payoff of $\delta^{t} u_{i}(x)$. The payoff of perpetual disagreement to any player is zero.

We shall restrict our attention to stationary strategies by which we mean the strategies such that (a) a proposal of any player is independent of the history of play and (b) the reaction of a player to a proposal only depends on the proposal itself. Thus a stationary strategy of player $i$ consists of a proposal $x_{i}$ and an acceptance set $A_{i}$. Player $i$ proposes the point $x_{i}$ whenever player $i$ becomes a proposer and he accepts a proposal $x$ if and only if $x$ is an element of the set $A_{i}$. Suppose now each player uses a stationary strategy. A joint stationary strategy $\sigma=\left(x_{\bullet}, A_{\bullet}\right)$ induces the acceptance set $A_{C}$ of the coalition $C$ and the social acceptance set $A$ as follows:

$$
A_{C}=\bigcap_{i \in C} A_{i} \text { and } A=\bigcup_{C \in \mathscr{C}} A_{C} .
$$

A proposal $x$ is implemented if and only if it is an element of the social acceptance set $A$. A joint stationary strategy $\sigma$ is a no-delay strategy if $x_{i} \in A$ for each player $i$. If such a strategy is being played, the first proposal to be made is implemented, so the game ends in period $t=0$. Thus the expected utility to player $i$ at the beginning of the game on the no-delay stationary strategy $\sigma$ is given by

$$
y_{i}=\sum_{k \in N} \mu(k) u_{i}\left(x_{k}\right)
$$

Definition 1 A joint stationary strategy $\sigma=\left(x_{\bullet}, A_{\bullet}\right)$ is a bargaining equilibrium of the game $\Gamma(\delta)$ if it is a no-delay strategy and for each $i \in N$ we have

$$
\begin{gathered}
x_{i}=\arg \max _{x \in A} u_{i}(x) \text { and } \\
A_{i}=\left\{x \in[0,1] \mid u_{i}(x) \geq \delta y_{i}\right\} .
\end{gathered}
$$

Each bargaining equilibrium is a subgame perfect equilibrium of the game $\Gamma(\delta)$. Conversely, given a subgame perfect equilibrium of $\Gamma(\delta)$ in stationary strategies satisfying an additional condition of weak dominance, there is a bargaining equilibrium having the same equilibrium proposals and inducing the same expected payoffs (see Banks and Duggan [1] and Cho and Duggan [5]).

Existence of bargaining equilibrium under the maintained assumptions follows from Theorems 1 and 2 in Banks and Duggan [1]. In general, there may be many bargaining equilibria, an example being given in Cho and Duggan [5]. Kalandrakis [12] gives an example of the game with a one-dimensional set of alternatives having a continuum of bargaining equilibria.

Sufficient conditions for the uniqueness of bargaining equilibrium are given in Cho and Duggan [5], Cardona and Ponsatí [4], Herings and Predtetchinski [11]. Thus Cho and 
Duggan [5] show that bargaining equilibrium is unique for each $\delta<1$ under the assumption that the utility functions $u_{i}$ are quadratic and the acceptance rule $\mathscr{C}$ is proper and strong. The acceptance rule $\mathscr{C}$ is said to be strong if for each $C \subset N$ either $C \in \mathscr{C}$ or $N \backslash C \in \mathscr{C}$. While any acceptance rule satisfying Assumption (A2) is necessarily proper, it does not have to be strong.

Cardona and Ponsatí [4] show that bargaining equilibrium is unique if recognition is deterministic, the players' utility functions are symmetric around the peak, and the quota acceptance rule is applied. Herings and Predtetchinski [11] establish the uniqueness of bargaining equilibria in a game with a Markov recognition process, assuming tent-shaped utility functions and the unanimity acceptance rule.

This contribution establishes instead the asymptotic uniqueness of bargaining equilibria. That is, we show that along any sequence of bargaining equilibria, as the discount factor goes to one, the social acceptance set converges to a limit which is uniquely determined by the primitives of the model.

\section{The social acceptance set}

This section establishes a number of preliminary results, some of which are known. We state them for the sake of completeness, as a preparation for the results of the following section. The main result of the section states that along any convergent sequence of bargaining equilibria of the game $\Gamma(\delta)$, as $\delta$ approaches one, the social acceptance set converges to a singleton set. We show by means of an example that Assumption (A2) plays a crucial role for this result.

Since we assume the utility functions to be concave and continuous, each individual acceptance set in a bargaining equilibrium is a closed interval. We write $\left[x_{i}^{-}, x_{i}^{+}\right]$to denote the individual acceptance set $A_{i}$ of player $i$. The acceptance set of coalition $C$ is also a closed interval denoted by $\left[x_{C}^{-}, x_{C}^{+}\right]$, where

$$
x_{C}^{-}=\max _{i \in C}\left\{x_{i}^{-}\right\} \text {and } x_{C}^{+}=\min _{i \in C}\left\{x_{i}^{+}\right\} .
$$

We write $E\left(x_{\bullet}\right)$ to denote the expected proposal, that is

$$
E\left(x_{\bullet}\right)=\sum_{i \in N} \mu(i) x_{i}
$$

Lemma 1 below is essentially Lemma 1 from Banks and Duggan [1]. To their result we add that the social acceptance set contains at least to distinct points.

Lemma 1 Let $\left(x_{\bullet}, A_{\bullet}\right)$ be a bargaining equilibrium of the game $\Gamma(\delta)$ inducing the social acceptance set $A$ and let $a=E\left(x_{\bullet}\right)$ denote the expected equilibrium proposal. Then $A$ is $a$ closed interval and

$$
[\delta a, 1-\delta+\delta a] \subset \bigcap_{i \in N} A_{i} \subset A
$$




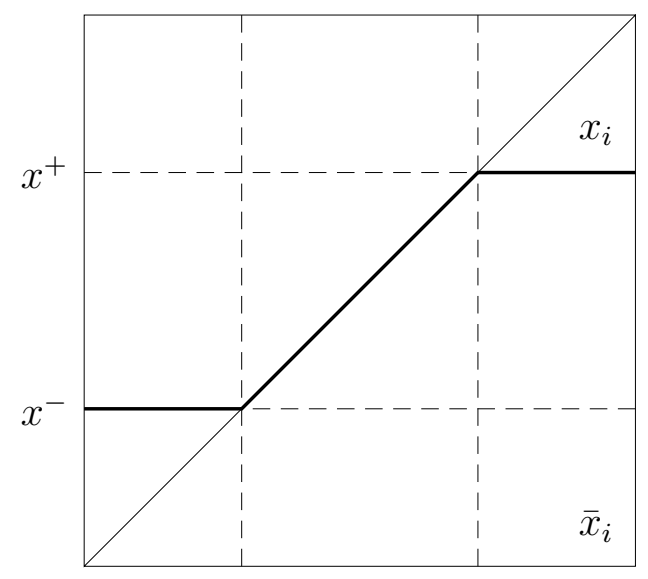

Figure 1: Equilibrium proposals.

Proof. Take any point $p$ in $[0,1]$. Using Jensen's inequality and the fact that $u_{i}(p) \geq 0$ we obtain the following chain of inequalities:

$$
u_{i}((1-\delta) p+\delta a) \geq(1-\delta) u_{i}(p)+\delta u_{i}(a) \geq \delta \sum_{k \in N} \mu(k) u_{i}\left(x_{k}\right)=\delta y_{i}
$$

This shows that the point $(1-\delta) p+\delta a$ is an element of the individual acceptance set $A_{i}$ of each player $i$. Since $p$ is an arbitrary point of $[0,1]$ we conclude that the entire interval $[\delta a, 1-\delta+\delta a]$ is contained in $A_{i}$ for each $i \in N$. This establishes the first inclusion. The second inclusion follows by the definition of the social acceptance set and because $\mathscr{C}$ contains the coalition $N$. Moreover, each point $(1-\delta) p+\delta a$ is an element of the acceptance set $A_{C}$ of each coalition $C$. Hence the social acceptance set is a closed interval, being a union of finitely many closed intervals $A_{C}$ having a point in common.

We let the social acceptance set be $\left[x_{-}, x_{+}\right]$, where

$$
x_{-}=\min _{C \in \mathscr{C}}\left\{x_{C}^{-}\right\} \text {and } x_{+}=\max _{C \in \mathscr{C}}\left\{x_{C}^{+}\right\}
$$

or equivalently

$$
x_{-}=\min _{C \in \mathscr{C}} \max _{i \in C}\left\{x_{i}^{-}\right\} \text {and } x_{+}=\max _{C \in \mathscr{C}} \min _{i \in C}\left\{x_{i}^{+}\right\} .
$$

The equilibrium proposal $x_{i}$ of player $i$ is a point of $\left[x_{-}, x_{+}\right]$closest to $\bar{x}_{i}$, the ideal point of individual $i$, thus

$$
x_{i}= \begin{cases}x_{-} & \text {if } \bar{x}_{i} \leq x_{-} \\ \bar{x}_{i} & \text { if } x_{-} \leq \bar{x}_{i} \leq x_{+} \\ x_{+} & \text {if } x_{+} \leq \bar{x}_{i}\end{cases}
$$

Figure 1 illustrates. 
Definition 2 Given a collection $\mathscr{C}$ of decisive coalitions, define the numbers $\alpha=\alpha(\mathscr{C})$ and $\beta=\beta(\mathscr{C})$ as follows:

$$
\alpha=\max _{C \in \mathscr{C}} \min _{i \in C}\left\{\bar{x}_{i}\right\} \text { and } \beta=\min _{C \in \mathscr{C}} \max _{i \in C}\left\{\bar{x}_{i}\right\}
$$

Lemma $2 \alpha \leq \beta$.

Proof. For let $C_{1} \in \mathscr{C}$ and $C_{2} \in \mathscr{C}$ be such that $\alpha=\min \left\{\bar{x}_{i}: i \in C_{1}\right\}$ and $\beta=\max \left\{\bar{x}_{i}\right.$ : $\left.i \in C_{2}\right\}$. Then by Assumption (A2) there is a player $i \in C_{1} \cap C_{2}$. Hence $\alpha \leq \bar{x}_{i} \leq \beta$.

It is not difficult to see that the interval $[\alpha, \beta]$ is exactly the core of the voting game as defined in Banks and Duggan [1]. As an example, consider a quota acceptance rule with the quota $q$. The collection $\mathscr{C}$ then consists of all coalitions $C \subset N$ with $|C| \geq q$. Let $N=\{1, \ldots, m\}$ and suppose that $\bar{x}_{1} \leq \cdots \leq \bar{x}_{m}$. Then $\alpha=\bar{x}_{m-q+1}$ and $\beta=\bar{x}_{q}$.

Lemma 3 Let $A=\left[x_{-}, x_{+}\right]$be the social acceptance set induced by a bargaining equilibrium of the game $\Gamma(\delta)$. Then $x_{-} \leq \beta$ and $\alpha \leq x_{+}$.

Proof. As before, let $\left[x_{i}^{-}, x_{i}^{+}\right]$be the individual acceptance set of player $i$. The point $\bar{x}_{i}$ is the maximum of the function $u_{i}$ on the set $[0,1]$, and so is an element of $A_{i}$. Thus $x_{i}^{-} \leq \bar{x}_{i} \leq x_{i}^{+}$for each $i \in N$. Using Equations (3.1) we obtain

$$
\begin{aligned}
& x_{-}=\min _{C \in \mathscr{C}} \max _{i \in C}\left\{x_{i}^{-}\right\} \leq \min _{C \in \mathscr{C}} \max _{i \in C}\left\{\bar{x}_{i}\right\}=\beta, \\
& x_{+}=\max _{C \in \mathscr{C}} \min _{i \in C}\left\{x_{i}^{+}\right\} \geq \max _{C \in \mathscr{C}} \min _{i \in C}\left\{\bar{x}_{i}\right\}=\alpha .
\end{aligned}
$$

Define

$$
S=\{i \in N \mid \mu(i)>0\} \text { and } \nu=\min _{i \in S}\{\mu(i)\} \text {. }
$$

Since $A$ is a closed interval in $[0,1]$ and the utility function $u_{i}$ is continuous, the set $u_{i}(A)$ is also a closed interval. We now show that the length of $u_{i}(A)$ in any bargaining equilibrium of the game $\Gamma(\delta)$ is bounded above by $(1-\delta) / \nu$ for at least one player $i \in S$. We write len $(I)$ for the length of an interval $I$, the difference of its upper and lower endpoints. Notice that assumption (A2) plays a crucial role in the proof of Lemma 4.

Lemma 4 Let $\left(x_{\bullet}, A_{\bullet}\right)$ be a bargaining equilibrium of the game $\Gamma(\delta)$ inducing the social acceptance set $A$. Then $\operatorname{len}\left(u_{i}(A)\right) \leq(1-\delta) / \nu$ for some $i \in S$.

Proof. Let $A=\left[x_{-}, x_{+}\right]$. There exist coalitions $C^{-}$and $C^{+}$in $\mathscr{C}$ such that $x_{-} \in A_{C^{-}}$and $x_{+} \in A_{C^{+}}$. Now, by assumption (A2) there exists a player $i \in C^{-} \cap C^{+}$with $\mu(i)>0$. Thus, both points $x_{-}$and $x_{+}$belong to the individual acceptance set $A_{i}$ of player $i$. Since $A_{i}$ is an interval, it contains the entire social acceptance set $A$. Fix one such player $i$. 
We can estimate the utility of player $i$ on the social acceptance set $A$ as follows. Since $A \subset A_{i}$, the utility of player $i$ on the set $A$ is bounded below by $\delta y_{i}$. On the other hand, the proposal $x_{i}$ maximizes player $i$ 's utility on the set $A$. Thus

$$
u_{i}(A) \subset\left[\delta y_{i}, u_{i}\left(x_{i}\right)\right] .
$$

We derive an upper bound on $u_{i}\left(x_{i}\right)$, as follows. The equilibrium proposal $x_{k}$ of each player $k$ is in the social acceptance set $A$, and consequently in the individual acceptance set $A_{i}$. Therefore, $u_{i}\left(x_{k}\right) \geq \delta y_{i}$ for each $k$. Thus,

$$
\begin{aligned}
y_{i} & =\sum_{k \in S} \mu(k) u_{i}\left(x_{k}\right) \\
& \geq \mu(i) u_{i}\left(x_{i}\right)+(1-\mu(i)) \delta y_{i} \\
& =\delta y_{i}+\mu(i)\left(u_{i}\left(x_{i}\right)-\delta y_{i}\right) \\
& \geq \delta y_{i}+\nu\left(u_{i}\left(x_{i}\right)-\delta y_{i}\right) \\
& =\nu u_{i}\left(x_{i}\right)+(1-\nu) \delta y_{i} .
\end{aligned}
$$

Rearranging yields the inequality

$$
u_{i}\left(x_{i}\right) \leq(1-\delta+\nu \delta) y_{i} / \nu
$$

Therefore,

$$
\operatorname{len}\left(u_{i}(A)\right) \leq u_{i}\left(x_{i}\right)-\delta y_{i} \leq(1-\delta+\nu \delta) y_{i} / \nu-\delta y_{i}=(1-\delta) y_{i} / \nu \leq(1-\delta) / \nu,
$$

where the last inequality follows from the fact that $y_{i} \leq 1$.

We are now in a position to prove the main result of this section: Along any convergent sequence of bargaining equilibria, as the discount factor converges to one, the social acceptance set collapses to a point. Theorem 1 can be obtained by combining Theorems 3 and 5 in Banks Duggan [1]. Instead we give a direct proof of the result, with the advantage of being able to exploit the special structure of one-dimensional bargaining.

Theorem 1 Let $A^{n}=\left[x_{-}^{n}, x_{+}^{n}\right]$ be the social acceptance set induced by some bargaining equilibrium of the game $\Gamma\left(\delta^{n}\right)$. If $\delta^{n} \longrightarrow 1, x_{-}^{n} \longrightarrow a_{-}$and $x_{+}^{n} \longrightarrow a_{+}$, then $a_{-}=a_{+}$.

Proof. Since $x_{-}^{n} \leq x_{+}^{n}$ for each $n$, in the limit $a_{-} \leq a_{+}$. Let $A=\left[a_{-}, a_{+}\right]$. Since $\operatorname{len}\left(u_{i}(I)\right)$ is continuous as a function of of the endpoints of the interval $I$, we have $\operatorname{len}\left(u_{i}\left(A^{n}\right)\right) \longrightarrow \operatorname{len}\left(u_{i}(A)\right)$. On the other hand, because the set $S$ is finite, the preceding lemma implies that there exists a player $i$ in $S$ such that $\operatorname{len}\left(u_{i}\left(A^{n}\right)\right) \leq\left(1-\delta^{n}\right) / \nu$ for each $n$. Hence len $\left(u_{i}\left(A^{n}\right)\right) \longrightarrow 0$. We conclude that $\operatorname{len}\left(u_{i}(A)\right)=0$, which means that the function $u_{i}$ is constant on the interval $A$. But under Assumption (A1) the function $u_{i}$ is non-constant on any non-degenerate interval. It follows that $A$ is a singleton set. Therefore, $a_{-}=a_{+}$, as desired.

Theorem 1 justifies the following definition. 
Definition 3 Let $\left[x_{-}^{n}, x_{+}^{n}\right]$ be the social acceptance set induced by some bargaining equilibrium of the game $\Gamma\left(\delta^{n}\right)$. Suppose that the sequence $\delta^{n}$ converges to one and the sequences $x_{-}^{n}$ and $x_{+}^{n}$ converge to $x$. Then the alternative $x$ is called a limit of bargaining equilibria.

Theorem 2 below which establishes that each limit of bargaining equilibria is an element of $[\alpha, \beta]$ can be deduced by combining Theorems 3 and 5 in Banks and Duggan [1] and by noticing that $[\alpha, \beta]$ is the core of the voting game. In our one-dimensional setting, however, the result follows at once from Lemma 3. The median voter result of Corollary 1 is likewise due to Banks and Duggan [1] (see the discussion following Theorem 5). The result states that if the passing of a proposal requires an approval of it by the simple majority of the voters, then the limit of bargaining equilibria is the ideal point of the median player, i.e. that individual who divides the set of players into two coalitions of equal size, those with lower ideal points and those with higher ideal points.

Theorem 2 Each limit of bargaining equilibria lies in the interval $[\alpha, \beta]$.

Proof. Let $x$ be a limit of bargaining equilibria. Thus there exists a sequence $\delta^{n}$ converging to 1 and for each $n$ a bargaining equilibrium of the game $\Gamma\left(\delta^{n}\right)$ with the social acceptance set $\left[x_{-}^{n}, x_{+}^{n}\right]$ such that the sequences $x_{-}^{n}$ and $x_{+}^{n}$ converge to the point $x$. By the preceding lemma, $x_{-}^{n} \leq \beta$ and $\alpha \leq x_{+}^{n}$ for each $n$. Taking the limit, we obtain the inequalities $x \leq \beta$ and $\alpha \leq x$.

Corollary 1 Suppose $N=\{1, \ldots, 2 m+1\}$ and $\bar{x}_{1} \leq \bar{x}_{2} \leq \cdots \leq \bar{x}_{2 m} \leq \bar{x}_{2 m+1}$. Suppose the collection $\mathscr{C}$ consists of all coalitions $C \subset N$ with $|C| \geq m+1$. Then the point $\bar{x}_{m+1}$ is the unique limit of of bargaining equilibria.

Proof. The result follows once we notice that $\alpha=\bar{x}_{m+1}=\beta$.

We have thus shown that along a converging sequence of bargaining equilibria the social acceptance set collapses to a point. In the following section we establish that this point, called the limit of bargaining equilibria, is in fact independent of the choice of the sequence of equilibria, and is uniquely determined by the primitives of the model.

We conclude this section with two examples that highlight the role of Assumptions (A1) and (A2) for the results of the paper. We first show that Theorem 1 need not be true if assumption (A2) is violated. Suppose there are three players $l, m$ and $r$, and the yes-votes of any two players are sufficient for a passing of a proposal. Assume that $\mu(l)=\mu(r)=1 / 2$ so that only players $l$ and $r$ ever make proposals. Assumption (A2) is violated because both $\{l, m\}$ and $\{m, r\}$ are decisive coalitions, but their intersection consists of player $m$ alone who has zero recognition probability.

Suppose that the utility function of player $l$ is $u_{l}(x)=1-x$, that of player $r$ is $u_{r}(x)=x$ and that of player $m$ is $u_{m}(x)=1-|x-1 / 2|$. The utility functions are illustrated in Figure 2. Assumption (A1) is clearly satisfied. 


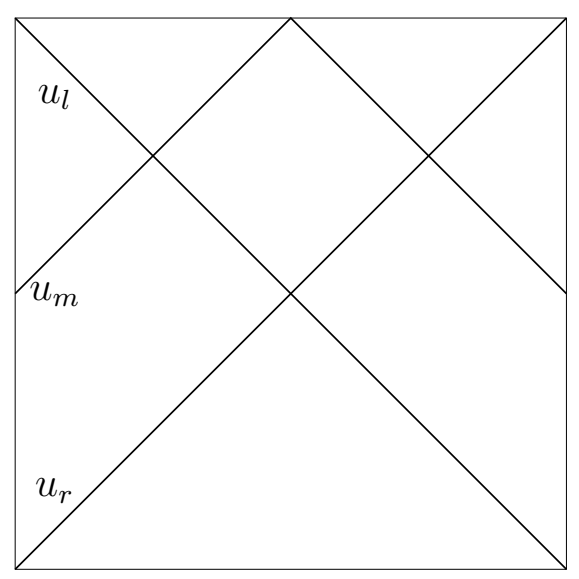

Figure 2: The utility functions in the example of Section 3.

Now, for each $\delta \in[0,1)$ there exists a bargaining equilibrium where $[0,1]$ is the social acceptance set. In this equilibrium player $l$ proposes the point 0 and player $r$ proposes the point 1 . The expected utility is $1 / 2$ for all players. The individual acceptance set of player $l$ is $A_{l}=[0,1-(\delta / 2)]$, the individual acceptance set of player $r$ is $A_{r}=[\delta / 2,1]$ and that of player $m$ is $[0,1]$. The acceptance set of the coalition $\{l, m\}$ equals $[0,1-(\delta / 2)]$, while the acceptance set of coalition $\{m, r\}$ equals $[\delta / 2,1]$. The union of $[0,1-(\delta / 2)]$ and $[\delta / 2,1]$ is clearly the entire set $[0,1]$.

Assumption (A1) requires the utility functions to be single-peaked and concave. We now show by means of the following example that uniqueness of the limit of bargaining equilibria breaks down if we drop the assumption of concavity of the utility functions. Suppose there are three players, 0,1 , and 2, with the utility functions $u_{0}(x)=e^{-x}$, $u_{1}(x)=e^{x-1}$ and $u_{2}(x)=\left(6-e^{1-x}\right) / 5$. The function $u_{0}$ is decreasing while $u_{1}$ and $u_{2}$ are increasing. Hence all three functions are strictly quasi-concave and single-peaked, but only $u_{2}$ is concave.

Suppose player 0 makes a proposal with the probability of $1 / 2$ while players 1 and 2 with the probability of $1 / 4$ each. Assume the unanimity rule.

Let $s_{\delta}=\ln (2-\delta)-\ln (\delta)$. Fix a point $\left.x \in\right] 0,1\left[\right.$ and let $\delta_{x}=2 /\left(1+e^{1-x}\right)$. The interval $\left[x, x+s_{\delta}\right]$ is the social acceptance set in a bargaining equilibrium of the game $\Gamma(\delta)$ whenever $\delta>\delta_{x}$. In equilibrium, player 0 proposes the point $x$ while the players 1 and 2 propose the point $x+s_{\delta}$. To see that this is indeed an equilibrium observe that player 0 is exactly indifferent between accepting and rejecting the point $x+s_{\delta}$, player 1 is exactly indifferent between accepting and rejecting the point $x$, while player 2 is (weakly) better off accepting $x$. That is $u_{0}\left(x+s_{\delta}\right)=\delta y_{0}$ and $u_{1}(x)=\delta y_{1}$ and $u_{2}(x) \geq \delta y_{2}$ where $y_{i}=\left[u_{i}(x)+u_{i}\left(x+s_{\delta}\right)\right] / 2$. Thus the individual acceptance sets for the players 0 and 1 are $A_{0}=\left[0, x+s_{\delta}\right]$ and $A_{1}=[x, 1]$, respectively, while player 2's acceptance set is a subset of $A_{1}$.

As $\delta$ converges to 1 the set $\left[x, x+s_{\delta}\right]$ converges to the singleton set $\{x\}$. Hence each point of $] 0,1[$ is a limit of bargaining equilibria. 


\section{The characteristic function}

We have so far shown that along a convergent sequence of bargaining equilibria as the discount factor approaches one, the social acceptance set collapses to a point. Any such point is called a limit of bargaining equilibria. In this section we define the characteristic function and show that its unique generalized zero point is an element of the social acceptance set of each bargaining equilibrium of the game $\Gamma(\delta)$ for each $\delta \in[0,1[$. As a consequence, the generalized zero of the characteristic function is the unique limit of bargaining equilibria.

The formulae of this section make use of the the left and right derivatives of the utility functions. We do not want to impose the differentiability of the utility functions by assumption because this would rule out the utilities that depend on the absolute distance of the alternative from the bliss point (as in Figure 2), which appears to be a natural choice for the utility functions.

Remark 1 Each function $u_{i}$ has left and right derivatives at each point $\left.x \in\right] 0,1$ [ denoted by $l_{i}(x)$ and $r_{i}(x)$. The function $u_{i}$ is differentiable at the point $\left.x \in\right] 0,1$ [ if and only if $r_{i}(x)=l_{i}(x)$. Both $l_{i}$ and $r_{i}$ are non-increasing functions on $] 0,1\left[\right.$ and $r_{i}(x) \leq l_{i}(x)$. The interval $\left[r_{i}(x), l_{i}(x)\right]$ is the subgradient of $u_{i}$ at point $x$. Given $\left.a \in\right] 0,1[$ and $x \in[0,1]$, the inequality $u_{i}(x)-u_{i}(a) \leq s(x-a)$ holds for each $s \in\left[r_{i}(a), l_{i}(a)\right]$. Since $\bar{x}_{i}$ is the unique maximum of $u_{i}$ on $[0,1]$ we have $0<r_{i}(x) \leq l_{i}(x)$ whenever $x<\bar{x}_{i}, r_{i}\left(\bar{x}_{i}\right) \leq 0 \leq l_{i}\left(\bar{x}_{i}\right)$, and $r_{i}(x) \leq l_{i}(x)<0$ whenever $\bar{x}_{i}<x$.

For each alternative $x$ define

$$
\mu_{-}(x)=\mu\left(\left\{i \in N \mid \bar{x}_{i} \leq x\right\}\right) \text { and } \mu_{+}(x)=\mu\left(\left\{i \in N \mid x<\bar{x}_{i}\right\}\right) .
$$

Thus $\mu_{-}(x)$ is the total recognition probability of the players whose ideal point lies in the interval $[0, x]$, while $\mu_{+}(x)$ is the complementary probability. The function $\mu_{-}$is the cumulative distribution function of the players' bliss points induced by the recognition probabilities. For each $x \in] 0,1[$ we define

$$
\begin{gathered}
\xi_{-}(x)=\max _{C \in \mathscr{C}} \min _{i \in C}\left\{r_{i}(x) / u_{i}(x)\right\} \text { and } \xi_{+}(x)=\min _{C \in \mathscr{C}} \max _{i \in C}\left\{l_{i}(x) / u_{i}(x)\right\}, \\
\xi(x)=\mu_{-}(x) \xi_{-}(x)+\mu_{+}(x) \xi_{+}(x) .
\end{gathered}
$$

The expressions is (4.2) are well defined, since each function $u_{i}$ is positive on $] 0,1[$. The function $\xi$ is referred to as a characteristic function. It will be convenient to extend the characteristic function to all of the interval $[0,1]$ by letting $\xi(0)=+\infty$ and $\xi(1)=-\infty$.

Lemma 5 The characteristic function $\xi$ is a decreasing function on $[0,1]$. We have $\xi_{-}(x) \leq \xi_{+}(x)$ for each $\left.x \in\right] 0,1[$.

Proof. Take an $x \in] 0,1\left[\right.$. Let $C_{-}$and $C_{+}$in $\mathscr{C}$ be such that

$$
\xi_{-}(x)=\min _{k \in C_{-}}\left\{r_{k}(x) / u_{k}(x)\right\} \text { and } \xi_{+}(x)=\max _{k \in C_{+}}\left\{l_{k}(x) / u_{k}(x)\right\},
$$


By Assumption (A2) the coalitions $C_{-}$and $C_{+}$have a non-empty intersection. For each $i \in C_{-} \cap C_{+}$we have

$$
\xi_{-}(x) \leq r_{i}(x) / u_{i}(x) \leq l_{i}(x) / u_{i}(x) \leq \xi_{+}(x) .
$$

Now $l_{k}(x) / u_{k}(x)$ and $r_{k}(x) / u_{k}(x)$ are the left and the right derivatives, respectively, of the strictly concave function $\ln u_{k}$ and hence are decreasing as functions of $x$ on the open interval $] 0,1\left[\right.$. It follows that both $\xi_{+}$and $\xi_{-}$are decreasing functions on $] 0,1[$. To see that the function $\xi$ is a decreasing function rewrite (4.3) as

$$
\xi(x)=\sum \mu(i) \xi_{i}(x), \text { where } \xi_{i}(x)= \begin{cases}\xi_{+}(x) & \text { if } x<\bar{x}_{i} \\ \xi_{-}(x) & \text { if } \bar{x}_{i} \leq x\end{cases}
$$

It is $\xi_{i}$ is a decreasing function for each $i$. The result follows.

The most important result of the paper is the following theorem.

Theorem 3 Let $A=\left[x_{-}, x_{+}\right]$be the social acceptance set induced by a bargaining equilibrium of the game $\Gamma(\delta)$. Then $\xi\left(x_{-}\right) \geq 0 \geq \xi\left(x_{+}\right)$.

The proof of Theorem 3 relegated to the Appendix.

Definition 4 The point $x \in[a, b]$ is a generalized zero of the function $f:[a, b] \rightarrow \mathbb{R} \cup$ $\{-\infty,+\infty\}$ if there are sequences $\left\{x_{-}^{n}\right\}$ and $\left\{x_{+}^{n}\right\}$ of points in $[a, b]$ converging to $x$ such that $\lim f\left(x_{-}^{n}\right) \leq 0 \leq \lim f\left(x_{+}^{n}\right)$. The point $x \in[a, b]$ is a generalized fixed point of the function $g:[a, b] \rightarrow[a, b]$ if it is a generalized zero of the function $f:[a, b] \rightarrow \mathbb{R}$ given by $f(x)=g(x)-x$.

The limits of the sequences $f\left(x_{-}^{n}\right)$ and $f\left(x_{+}^{n}\right)$ in the above definition are understood to be the limits in the extended real line and are allowed to be infinite.

It is clear that any point $x \in[a, b]$ such that $f(x)=0$ is a generalized zero of the function $f$ (take $x_{-}^{n}=x$ and $x_{+}^{n}=x$ ). Conversely, if $x$ is a generalized zero of $f$ and $f$ is continuous at $x$, then $f(x)=0$. A decreasing function $f$ has at most one generalized zero. A decreasing function $f$ such that $f(a) \geq 0 \geq f(b)$ has exactly one generalized zero. Moreover, the point $p$ is a generalized zero of such a function $f$ if and only if $f(x)>0$ for each $x$ in $[a, b]$ with $x<p$ and $f(x)<0$ for each $x$ in $[a, b]$ with $p<x$.

The inequalities of Theorem 3 can be restated as $x_{-} \leq p \leq x_{+}$where $p$ denotes the generalized zero of the function $\xi$.

Theorem 4 The limit of bargaining equilibria is unique and is equal to the (unique) generalized zero of the function $\xi$.

Proof. Let $x$ be a limit of bargaining equilibria. By Definition 3, there exist sequences $x_{-}^{n}$, $x_{+}^{n}$ and $\delta^{n}$ such that $\left[x_{-}^{n}, x_{+}^{n}\right]$ is the social acceptance set in a bargaining equilibrium of the game $\Gamma\left(\delta^{n}\right)$, the sequence $\delta^{n}$ converges to 1 and both $x_{-}^{n}$ and $x_{+}^{n}$ converge to $x$. We have 
$\xi\left(x_{-}^{n}\right) \geq 0 \geq \xi\left(x_{+}^{n}\right)$ for every $n$ by Theorem 3 . Hence $\lim \xi\left(x_{-}^{n}\right) \geq 0 \geq \lim \xi\left(x_{+}^{n}\right)$. Thus the point $x$ is a generalized zero of the function $\xi$.

The following result follows easily from the preceding theorems.

Theorem 5 Let $x$ be the limit of bargaining equilibria. Let $0 \leq \delta<1$ and let $A$ be the social acceptance set in a bargaining equilibrium of $\Gamma(\delta)$. Then $x$ is an element of $A$.

We now discuss an alternative definition for the characteristic function and provide some intuition behind our results. Recall that by Theorem 2 each limit of bargaining equilibria lies in the interval $[\alpha, \beta]$. In particular, if $\alpha$ coincides with $\beta$, as under the simple majority voting rule for example, then $\alpha=\beta$ is the unique of limit bargaining equilibria. Thus suppose $\alpha<\beta$ and for each point $x \in] \alpha, \beta[$ define

$$
\varphi_{-}(x)=\min _{C \in \mathscr{C}} \max _{\substack{i \in C \\ x<\bar{x}_{i}}}\left\{-\frac{u_{i}(x)}{l_{i}(x)}\right\} \text { and } \varphi_{+}(x)=\max _{C \in \mathscr{C}} \min _{\substack{i \in C \\ \bar{x}_{i}<x}}\left\{-\frac{u_{i}(x)}{r_{i}(x)}\right\} .
$$

Notice that the expressions in (4.4) involve no division by zero since $l_{i}(x)>0$ whenever $x<\bar{x}_{i}$ and $r_{i}(x)<0$ whenever $\bar{x}_{i}<x$. Furthermore, the maxima and the minima are always taken over non-empty sets: since $\alpha<x<\beta$ each coalition $C \in \mathscr{C}$ contains some player $i$ with $x<\bar{x}_{i}$ and some player $k$ with $\bar{x}_{k}<x$. Notice that $\varphi_{-}(x)<0<\varphi_{-}(x)$ for each $x \in] \alpha, \beta[$. Define

$$
\varphi(x)=\mu_{-}(x) \varphi_{-}(x)+\mu_{+}(x) \varphi_{+}(x)
$$

for each $\alpha<x<\beta$. We extend $\varphi$ it to all of the interval $[0,1]$ by letting $\varphi(x)=+\infty$ for $x \leq \alpha$ and $\varphi(x)=-\infty$ for $\beta \leq x$.

All our results could be restated in terms of the function $\varphi$. Indeed, the function $\varphi$ is decreasing on the interval $[\alpha, \beta]$ and its generalized zero equals that of the function $\xi$. To see this notice that for each $x \in] \alpha, \beta[$ we have

$$
\varphi_{-}(x)=-1 / \xi_{+}(x) \text { and } \varphi_{+}(x)=-1 / \xi_{-}(x) .
$$

Hence, for each $x \in[0,1]$ we have $\xi(x)<0$ if and only if $\varphi(x)<0$ and $\xi(x)>0$ if and only if $\varphi(x)>0$. Thus both Theorems 3 and 4 continue to be true if $\xi$ is replaced by $\varphi$.

What is the intuition behind these definitions? Let us make the following mental exercise: Take a bargaining equilibrium with the expected equilibrium proposal $a=E\left(x_{\bullet}\right)$ and consider replacing the utility function of player $i$ in the definition of bargaining equilibrium by its first-order approximation $u_{i}(x) \approx u_{i}(a)+u_{i}^{\prime}(a)(x-a)$ around the point $a$. The expected utility to player $i$ can then be approximated as $y_{i} \approx u_{i}(a)$. Recall now that player $i$ accepts the proposal $x$ if and only if $u_{i}(x) \geq \delta y_{i}$, the condition that can be approximated as $u_{i}^{\prime}(a)(x-a) \geq-(1-\delta) u_{i}(a)$. Hence we find that the left endpoint (if $u_{i}^{\prime}(a)>0$ ) or the right endpoint (if $u_{i}^{\prime}(a)<0$ ) of the individual acceptance set $A_{i}$ of player $i$ is approximately given by $a-(1-\delta) u_{i}(a) / u_{i}^{\prime}(a)$.

Now suppose that no ideal point falls inside the social acceptance set. Thus for each player $i$ either $\bar{x}_{i}<x_{-}$or $x_{+}<\bar{x}_{i}$. Using Equations (3.1) we approximate the endpoints 


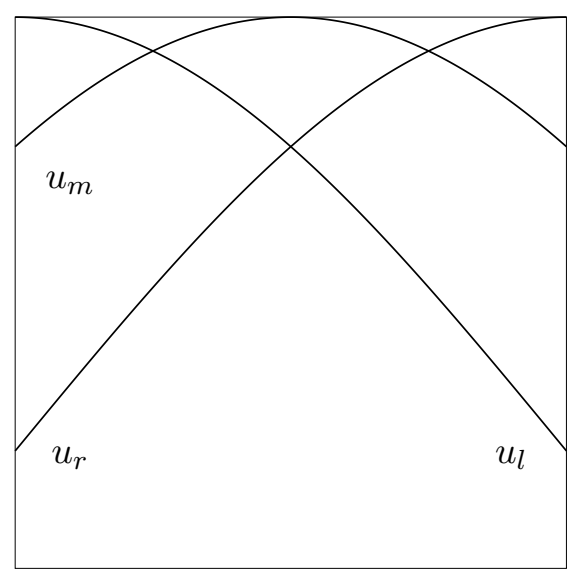

Figure 3: The utility functions (4.6), where $\bar{x}_{l}=0, \bar{x}_{m}=1 / 2$, and $\bar{x}_{r}=1$ (The horizontal axis extends from 0 to 1 , the vertical axis from 0.5 to 1 ).

of the social acceptance set. It is easy to see that when taking the maximum of $x_{i}^{-}$over the players $i$ in some coalition $C$ we can disregard the players with $\bar{x}_{i}<x_{-}$. Similarly, when taking the minimum of $x_{i}^{+}$over $i \in C$ we can disregard the players with $x_{+}<\bar{x}_{i}$. This yields the approximations $x_{-} \approx a+(1-\delta) \varphi_{-}(a)$ and $x_{+} \approx a+(1-\delta) \varphi_{+}(a)$. Or equivalently,

$$
\frac{x_{-}-a}{1-\delta} \approx \varphi_{-}(a) \text { and } \frac{x_{+}-a}{1-\delta} \approx \varphi_{+}(a)
$$

Thus $\varphi_{-}(a)$ and $\varphi_{+}(a)$ can be interpreted as the he coefficients in the first-order Taylor expansion of the endpoints of the social acceptance set. The difference $\varphi_{+}(a)-\varphi_{-}(a)$ is the speed of convergence of the length $x_{+}-x_{-}$of the social acceptance set to zero.

Since no ideal point falls inside the social acceptance set, the point $x_{-}$is proposed with a probability of $\mu_{-}(a)$ and the point $x_{+}$with the probability of $\mu_{+}(a)$ so that the expected proposal is $a=\mu_{-}(a) x_{-}+\mu_{+}(a) x_{+}$. Substituting the first-order approximations for $x_{-}$ and $x_{+}$we arrive at the condition that $\varphi(a)$ be approximately zero.

We conclude this section with a number of special cases and examples. Consider the utility functions given by the equation

$$
u_{i}(x)=\exp \left(-\left(x-\bar{x}_{i}\right)^{2} / 2\right) .
$$

Figure 3 depicts the utility functions (4.6) with the peaks at $0,1 / 2$ and 1 . We have $u_{i}^{\prime}(x)=u_{i}(x)\left(\bar{x}_{i}-x\right)$ and $u_{i}^{\prime \prime}(x)=-u_{i}(x)\left[1-\left(\bar{x}_{i}-x\right)^{2}\right]$. Since both $x$ and $\bar{x}_{i}$ are in $[0,1]$ we have $\left(\bar{x}_{i}-x\right)^{2} \leq 1$ and therefore $u_{i}^{\prime \prime}(x) \leq 0$. Thus the functions $u_{i}$ are concave on $[0,1]$. We have the following result.

Corollary 2 Suppose the utility functions are given by Equation (4.6). Then the limit of bargaining equilibria is a generalized fixed point of the function $f$ given by $f(x)=$ $\beta-(\beta-\alpha) \mu_{-}(x)$. 
Proof. Since the utility functions are differentiable, the left and the right derivatives at each point coincide with the derivative denoted by $u_{i}^{\prime}(x)$. Now $u_{i}^{\prime}(x) / u_{i}(x)=-x+\bar{x}_{i}$. It follows that

$$
\begin{aligned}
& \xi_{-}(x)=\max _{C \in \mathscr{C}} \min _{i \in C}\left\{-x+\bar{x}_{i}\right\}=-x+\max _{C \in \mathscr{C}} \min _{i \in C}\left\{\bar{x}_{i}\right\}=-x+\alpha \\
& \xi_{+}(x)=\min _{C \in \mathscr{C}} \max _{i \in C}\left\{-x+\bar{x}_{i}\right\}=-x+\min _{C \in \mathscr{C}} \max _{i \in C}\left\{\bar{x}_{i}\right\}=-x+\beta .
\end{aligned}
$$

Therefore

$$
\xi(x)=-x+\alpha \mu_{-}(x)+\beta \mu_{+}(x)=-x+f(x) .
$$

The result follows.

We now illustrate by means of an example that the limit of bargaining equilibria does not in general coincide with the asymmetric Nash bargaining solution. Define the asymmetric Nash bargaining solution as a maximizer of the asymmetric Nash product $\rho(x)=\times u_{i}(x)^{\mu_{i}}$ on $[0,1]$, where the product is taken over the players $i \in N$ with $\mu(i)>0$. For the utility functions given by Equation (4.6) the logarithm of the asymmetric Nash product is given by $\ln \rho(x)=\sum \mu(i)\left(x-\bar{x}_{i}\right)^{2} / 2$. Clearly the asymmetric Nash bargaining solution is the average ideal point $\sum \mu(i) \bar{x}_{i}$.

Now suppose there are three players with equal recognition probabilities whose ideal points are $0, p$ and 1 . Suppose the approval of all three players is needed for the passing of a proposal. Then $\alpha=0, \beta=1$ and so the limit of bargaining equilibria is the generalized fixed point of the function $\mu_{+}$. Hence the limit of bargaining equilibria equals the generalized fixed point of the function $\mu_{+}$, which one can easily see to be

$$
\begin{cases}1 / 3 & \text { if } 0 \leq p \leq 1 / 3 \\ p & \text { if } 1 / 3 \leq p \leq 2 / 3 \\ 2 / 3 & \text { if } 2 / 3 \leq p \leq 1\end{cases}
$$

On the other hand the asymmetric Nash bargaining solution is equal to $(p+1) / 3$ and is not equal to the limit of bargaining equilibria unless $p=1 / 2$.

Two of our results below are motivated by the setup of the model in Herings and Predtetchinski [11], where the players are assumed to have tent-shaped utility functions $u_{i}(x)=1-\left|x-\bar{x}_{i}\right|$. In addition Herings and Predtetchinski [11] assume that there is a player with a peak at point 0 and a player with a peak at 1 , and consider only the unanimity acceptance rule. The focus of [11] is somewhat different from ours: the authors concentrate on the analysis of a class of Markov recognition rules. For the case of timeinvariant recognition the authors show (see Proposition 7.4) that the limit of bargaining equilibrium equals the generalized fixed point of the function $\mu_{+}$.

Using Theorem 4 we extend this result in two ways: In the next section we derive the bargaining equilibria under the tent-shaped utility functions for the general acceptance rule and the distribution of bliss points. Below we retain the unanimity acceptance rule but, rather than assuming that all players have a tent-shaped utility function, we require 
that there be two players, called $a$ and $b$, having linear utility functions $u_{a}(x)=1-x$ and $u_{b}(x)=x$.

Corollary 3 Suppose $\mathscr{C}=\{N\}$. Suppose $u_{a}(x)=1-x$ for some player $a \in N$ and $u_{b}(x)=x$ for some player $b \in N$. Then the limit of bargaining equilibria equals the generalized fixed point of the function $\mu_{+}$.

Proof. Let $0<x<1$. Using the fact that the functions $u_{i}$ are concave and non-negative we obtain the inequalities

$$
\begin{aligned}
& -u_{i}(x) \leq u_{i}(0)-u_{i}(x) \leq l_{i}(x)(0-x) \\
& -u_{i}(x) \leq u_{i}(1)-u_{i}(x) \leq r_{i}(x)(1-x)
\end{aligned}
$$

Rearranging these yields

$$
-1 /(1-x) \leq r_{i}(x) / u_{i}(x) \leq l_{i}(x) / u_{i}(x) \leq 1 / x
$$

It follows that

$$
\begin{aligned}
& \xi_{-}(x)=\min _{i \in N}\left\{r_{i}(x) / u_{i}(x)\right\}=u_{a}^{\prime}(x) / u_{a}(x)=-1 /(1-x) \\
& \xi_{+}(x)=\max _{i \in N}\left\{l_{i}(x) / u_{i}(x)\right\}=u_{b}^{\prime}(x) / u_{b}(x)=1 / x .
\end{aligned}
$$

The value of the function $\xi$ at $x$ is therefore given by

$$
\xi(x)=-\frac{\mu_{-}(x)}{1-x}+\frac{\mu_{+}(x)}{x}=\frac{\mu_{+}(x)-x}{x(1-x)} .
$$

Let $f(x)=\mu_{+}(x)-x$. For each $x \in[0,1]$ we have $\xi(x) \geq 0$ if and only if $f(x) \geq 0$ and $\xi(x) \leq 0$ if and only if $f(x) \leq 0$. Therefore if the point $p$ is a generalized zero of the function $\xi$ it is also a generalized zero of the function $f$ and hence a generalized fixed point of the function $\mu_{+}$.

\section{Some implications}

In this section we consider the families of utility functions satisfying the following assumption.

(A3) There exists a concave decreasing and continuously differentiable function $h:[0,1] \rightarrow$ $[0,1]$ such that $u_{i}(x)=h\left(\left|x-\bar{x}_{i}\right|\right)$ for each $i \in N$.

We refer to the functions satisfying this assumption as symmetric because the utility function of player $i$ can be obtained from that of player $k$ by shifting it horizontally by $\bar{x}_{i}-\bar{x}_{k}$. Moreover, each player's utility function is symmetric around its peak. 
Notice that the utility functions satisfying condition (A3) necessarily satisfy condition (A1). One interesting family of utility functions satisfying (A3) are the tent-shaped utilities given by

$$
u_{i}(x)=1-\left|x-\bar{x}_{i}\right|,
$$

in which case the function $h$ is given by $h(x)=1-x$. The utility functions given by (4.6) is another useful special case of (A3) (in this case $h(x)=\exp \left(-x^{2} / 2\right)$ ).

A symmetric family of utility functions offers a natural setup for the study of comparative statics of the limit of bargaining equilibria with respect to the acceptance rule. The reason for this is the way the acceptance rule $\mathscr{C}$ enters the characteristic equation: as is clear from the formulae below the function $\xi$ only depends on the collection $\mathscr{C}$ through the variables $\alpha(\mathscr{C})$ and $\beta(\mathscr{C})$. These variables therefore summarize all relevant aspects of the acceptance rule.

Lemma 6 Suppose the assumption (A3) holds. Let $0<x<1$ and $v(x)=h^{\prime}(x) / h(x)$. We have

$$
\xi(x)= \begin{cases}-\mu_{-}(x) v(\alpha-x)-\mu_{+}(x) v(\beta-x) & \text { if } x<\alpha \\ +\mu_{-}(x) v(x-\alpha)-\mu_{+}(x) v(\beta-x) & \text { if } \alpha \leq x \leq \beta \\ +\mu_{-}(x) v(x-\alpha)+\mu_{+}(x) v(x-\beta) & \text { if } \beta<x\end{cases}
$$

Proof. Define the functions $f_{-}$and $f_{+}$by the following equations:

$$
f_{-}(x)=\left\{\begin{array}{ll}
-v(-x) & \text { if } x<0 \\
v(x) & \text { if } 0 \leq x,
\end{array} \quad f_{+}(x)= \begin{cases}-v(-x) & \text { if } x \leq 0 \\
v(x) & \text { if } 0<x\end{cases}\right.
$$

Then

$$
r_{i}(x) / u_{i}(x)=f_{-}\left(x-\bar{x}_{i}\right) \text { and } l_{i}(x) / u_{i}(x)=f_{+}\left(x-\bar{x}_{i}\right) .
$$

The function $v$ is decreasing, because it is the derivative of the logarithm of the function $h$, and it is everywhere non-positive, because $h$ is decreasing. It follows that the functions $f_{-}$and $f_{+}$are decreasing. Hence, the expressions $f_{-}\left(x-\bar{x}_{i}\right)$ and $f_{+}\left(x-\bar{x}_{i}\right)$ are increasing in $\bar{x}_{i}$ for each $x$. Therefore

$$
\begin{aligned}
& \xi_{-}(x)=\max _{C \in \mathscr{C}} \min _{i \in C}\left\{f_{-}\left(x-\bar{x}_{i}\right)\right\}=f_{-}\left(x-\max _{C \in \mathscr{C}} \min _{i \in C}\left\{\bar{x}_{i}\right\}\right)=f_{-}(x-\alpha) \\
& \xi_{+}(x)=\min _{C \in \mathscr{C}} \max _{i \in C}\left\{f_{+}\left(x-\bar{x}_{i}\right)\right\}=f_{+}\left(x-\min _{C \in \mathscr{C}} \max _{i \in C}\left\{\bar{x}_{i}\right\}\right)=f_{+}(x-\beta) .
\end{aligned}
$$

Hence

$$
\xi(x)=\mu_{-}(x) f_{-}(x-\alpha)+\mu_{+}(x) f_{+}(x-\beta) .
$$

The result follows.

As is clear from these expressions, the limit of bargaining equilibria under Assumption (A3) depends on the acceptance rule $\mathscr{C}$ only through the variables $\alpha(\mathscr{C})$ and $\beta(\mathscr{C})$. Corollary 4 below shows it is a non-decreasing function of both. 
Corollary 4 Suppose Assumption (A3) is satisfied. Then the limit of bargaining equilibria is non-decreasing as a function of $\alpha$ and non-decreasing as a function of $\beta$.

Proof. Consider Equation (5.3). Since both functions $f_{-}$and $f_{+}$are decreasing, $\xi(x)$ is non-decreasing in $\alpha$ and non-decreasing in $\beta$ for each fixed $x \in] 0,1[$. The result now follows since $\xi(x)$ is decreasing in $x$.

Using Lemma 6 we derive the limit of bargaining equilibria for the tent-shaped utility functions.

Corollary 5 Suppose the utility functions are given by Equation (5.1). Then the limit of bargaining equilibria is equal to $\alpha$ if

$$
\frac{1}{2+\alpha-\beta} \leq \mu_{-}(\alpha)
$$

it is equal to $\beta$ if

$$
\mu_{-}(\beta) \leq \frac{1+\alpha-\beta}{2+\alpha-\beta}
$$

and it is equal to the generalized fixed point of the function $g:[\alpha, \beta] \rightarrow[\alpha, \beta]$ given by $g(x)=1+\alpha-\mu_{-}(x)(2+\alpha-\beta)$ if

$$
\mu_{-}(\alpha) \leq \frac{1}{2+\alpha-\beta} \text { and } \frac{1+\alpha-\beta}{2+\alpha-\beta} \leq \mu_{-}(\beta) .
$$

Proof. We have $h(a)=1-a$ and $v(a)=-1 /[1-a]$. For each $x \in] \alpha, \beta[$ we have

$$
\xi(x)=\frac{-x+1+\alpha-\mu_{-}(x)[2+\alpha-\beta]}{(1-x+\alpha)(1+x-\beta)} .
$$

In the first case one can easily see that $\xi(x)<0$ for each $\alpha<x<\beta$. Since by Theorem 2 the limit of bargaining equilibria lies in the interval $[\alpha, \beta]$, it must be equal to $\alpha$. Similarly, in the second case $\xi(x)>0$ for each $\alpha<x<\beta$, so that $\beta$ is the generalized zero of $\xi$. Finally, consider the third case. Let the function $f:[\alpha, \beta] \rightarrow \mathbb{R}$ be given by $f(x)=g(x)-x$. It is easy to see that for each point $x \in] \alpha, \beta[$ we have $f(x)>0$ if and only if $\xi(x)>0$ and $f(x)<0$ if and only if $\xi(x)<0$. Therefore if the point $p \in[\alpha, \beta]$ is a generalized fixed point of the function $g$, and hence a generalized zero of the function $f$, it is also a generalized zero of the function $\xi$.

We now turn to the comparative statics of the limit of bargaining equilibria with respect to the acceptance rule. First we argue that, if the recognition probabilities are uniform and the utility functions are symmetric, the outcome of bargaining under the unanimity acceptance rule is in some sense less extreme than that under the simple majority rule. Consider first an example where there are $m+1$ players with the utility functions $u_{0}(x)=$ $1-x$ and $m$ players with the utility functions $u_{1}(x)=x$. Each player has a recognition 
probability of $1 /(2 m+1)$. Since the point 0 is the ideal point of the median voter, 0 is the limit of bargaining equilibria under the simple majority rule. On the other hand, the limit of bargaining equilibria under the unanimity acceptance rule is $m /(2 m+1)$. Thus no matter how many players there are, the simple majority rule selects the extreme left of the interval $[0,1]$, while the unanimity acceptance rule leads to a more intermediate outcome. This feature of the example can be generalized as follows.

Let $a$ and $b$ denote the extreme left and the extreme right ideal points, respectively, that is

$$
a=\min _{i \in N}\left\{\bar{x}_{i}\right\} \text { and } b=\max _{i \in N}\left\{\bar{x}_{i}\right\} .
$$

Whatever the acceptance rule, the limit of bargaining equilibria always lies between these extreme points. Claim 1 establishes that the point selected by the unanimity acceptance rule is closer to the middle of the interval $[a, b]$ than the point selected by the simple majority rule.

Claim 1 Suppose Assumption (A3) is satisfied. Suppose $|N|=2 m+1$ and $\mu(i)=1 /(2 m+$ 1) for each $i \in N$. Let $x^{s}$ and $x^{u}$ denote the limit of bargaining equilibria under the simple majority rule and the unanimity acceptance rule, respectively. Let $c=(a+b) / 2$. If $x^{s} \leq c$ then $x^{s} \leq x^{u} \leq c$ and if $c \leq x^{s}$ then $c \leq x^{u} \leq x^{s}$.

Proof. Let $\xi$ be the characteristic function under the unanimity acceptance rule. The expression for $\xi$ is given by Lemma 6 , with $\alpha=a$ and $\beta=b$. Recall that the point $x^{u}$ is a generalized zero of the function $\xi$. To prove the claim it is sufficient to show that (A) if $x<x^{s}$ and $x<c$ then $\xi(x) \geq 0$, and (B) if $x>x^{s}$ and $x>c$ then $\xi(x) \leq 0$. It is easy to see that facts (A) and (B) indeed imply the claim.

Recall that if we label the players so that $\bar{x}_{1} \leq \cdots \leq \bar{x}_{2 m+1}$ then $x^{s}=\bar{x}_{m+1}$ (see Corollary 1). Because the recognition probabilities are assumed to be uniform, we have $1 / 2 \leq \mu_{-}\left(x^{s}\right)$ and $\mu_{-}(x) \leq 1 / 2$ whenever $x<x^{s}$.

To prove fact (A) take an $x$ in $[0,1]$ such that $x<x^{s}$ and $x<c$. If $x<a$ then $\xi(x) \geq 0$ as can be seen directly from (5.2) because the function $v$ is everywhere non-positive. Suppose $a \leq x$. Since $x<c<b$, we have

$$
\xi(x)=\mu_{-}(x) v(x-a)-\mu_{+}(x) v(b-x) \geq[v(x-a)-v(b-x)] / 2 \geq 0,
$$

where the first inequality holds because $\mu_{-}(x) \leq 1 / 2$ and $\mu_{+}(x) \geq 1 / 2$ for $x<x^{s}$. To see that the second inequality is true we notice that since $x<c$ we have $x-a \leq b-x$. Since $v$ is a decreasing function we have $v(x-a) \geq v(b-x)$. This proves fact $(\mathrm{A})$. The proof of fact $(\mathrm{B})$ is similar.

Claim 1 implies that the unanimity acceptance rule is superior to the simple majority rule according to the Rawlsian welfare criterion. Indeed, under (A3) the worst-off player is the player whose bliss point is most distant from the implemented alternative. Clearly, the most distant bliss point is either the point $a$ or the point $b$. The closer the alternative 


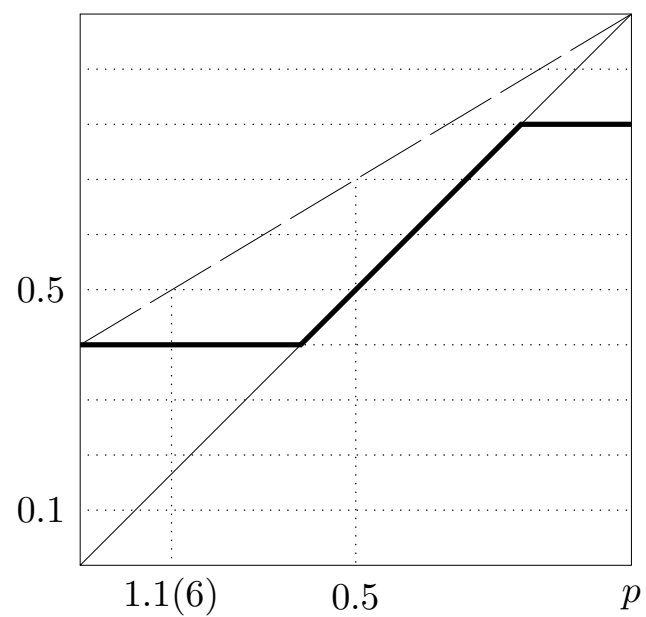

Figure 4: The limit of bargaining equilibria under different quota rules.

The limit of bargaining equilibrium is given by the diagonal if $q=3$, the dashed line if $q=4$ and the thick line if $q=5$.

to the midpoint $c$ of the interval $[a, b]$ the higher the utility of the worst-off player. Hence the maximization of the utility of the worst-off player dictates a choice of $x^{u}$ over $x^{s}$.

The quota rules can be more or less extreme than the unanimity rule depending on the distribution of the bliss points. Consider an example with five players, where the utility functions are given by Equation (4.6) and the recognition probability is $1 / 5$ for each player. Suppose one player has the ideal point 0 , two players the ideal point $p$ and two players the ideal point 1. Consider a quota acceptance rule that requires the approval of a proposal by at least $q$ players. Since the point $p$ is the ideal point the median player, it is the limit of bargaining equilibria if $q=3$. If $q=4$ the limit of bargaining equilibria is given by $(2+3 p) / 5$ and if $q=5$ it is given by

$$
\begin{cases}2 / 5 & \text { if } p \leq 2 / 5 \\ p & \text { if } 2 / 5 \leq p \leq 4 / 5 \\ 4 / 5 & \text { if } 4 / 5 \leq p\end{cases}
$$

Figure 4 depicts the limit of bargaining equilibria as a function of $p$ for each quota. In particular, if $p=1 / 2$, the limit of bargaining equilibria is $1 / 2$ if $q=3$ or $q=5$, but is $7 / 10$ if $q=4$. If $p=1 / 6$, the limit of bargaining equilibria is $1 / 6$ if $q=3,1 / 2$ if $q=4$ and $2 / 5$ if $q=5$. This example also shows that the limit of bargaining equilibria need not be a monotone function of the quota $q$.

Now consider altering the acceptance rule by removing or adding a decisive coalition. Let $\mathscr{C}$ and $\mathscr{C}^{\prime}$ be two acceptance rules and let $x(\mathscr{C})$ and $x\left(\mathscr{C}^{\prime}\right)$ be the corresponding limits of bargaining equilibria. We shall write $\min C$ and $\max C$ to denote the minimum and the maximum of the ideal points $\bar{x}_{k}$ of the players $k$ in the coalition $C$.

Claim 2 Let the collection $\mathscr{C}^{\prime}$ be obtained from $\mathscr{C}$ by adding the coalition $T$ and all its supersets, that is $\mathscr{C}^{\prime}=\mathscr{C} \cup\{C: T \subset C \subset N\}$. Suppose both $\mathscr{C}$ and $\mathscr{C}^{\prime}$ satisfy Assumption 
(A2). Then $x\left(\mathscr{C}^{\prime}\right) \leq x(\mathscr{C})$ if either $(a) \bar{x}_{i} \leq x(\mathscr{C})$ for each $i \in T$ or if (b) the utility functions satisfy Assumption (A3) and $\bar{x}_{k} \leq \alpha(\mathscr{C})$ for some player $k \in T$.

Proof. It is easy to see that $\alpha\left(\mathscr{C}^{\prime}\right)=\max \{\alpha(\mathscr{C}), \min T\}$ and $\beta\left(\mathscr{C}^{\prime}\right)=\min \{\beta(\mathscr{C}), \max T\}$. Under assumption (a) it holds that $\max T \leq x(\mathscr{C})$ and hence $\beta\left(\mathscr{C}^{\prime}\right) \leq x(\mathscr{C})$. Moreover, $x\left(\mathscr{C}^{\prime}\right) \leq \beta\left(\mathscr{C}^{\prime}\right)$ by Theorem 2. Under assumption (b) we have $\min T \leq \alpha(\mathscr{C})$ so that $\alpha(\mathscr{C})=\alpha\left(\mathscr{C}^{\prime}\right)$. Since $\beta\left(\mathscr{C}^{\prime}\right) \leq \beta(\mathscr{C})$ the result follows from Corollary 4 .

A coalition $T \in \mathscr{C}$ is said to be minimal if there is no $C \in \mathscr{C}$ with $C \subset T$ and $C \neq T$. Claim 3 shows that removing a minimal decisive coalition not containing player $i$ never makes player $i$ worse off.

Claim 3 Suppose the utility functions satisfy Assumption (A3). Let $T \in \mathscr{C}$ be a minimal decisive coalition let $\mathscr{C}^{\prime}=\mathscr{C} \backslash\{T\}$. Suppose the collections $\mathscr{C}$ and $\mathscr{C}^{\prime}$ satisfy Assumption (A2). Then $\left|x\left(\mathscr{C}^{\prime}\right)-\bar{x}_{i}\right| \leq\left|x(\mathscr{C})-\bar{x}_{i}\right|$ for each $i \in N \backslash T$.

Proof. Since $\mathscr{C}^{\prime} \subset \mathscr{C}$ we have $\alpha\left(\mathscr{C}^{\prime}\right) \leq \alpha(\mathscr{C})$ and $\beta(\mathscr{C}) \leq \beta\left(\mathscr{C}^{\prime}\right)$. Fix a player $i \in N \backslash T$. Since $T$ is an element of the collection $\mathscr{C}$ and since $\mathscr{C}$ is a monotone collection, the coalition $\{i\} \cup T$ is also an element of $\mathscr{C}$. Therefore $\{i\} \cup T$ is an element of $\mathscr{C}^{\prime}$, implying the following:

$$
\begin{aligned}
& \bar{x}_{i} \leq \max T \Rightarrow \max (\{i\} \cup T)=\max T \quad \Rightarrow \beta(\mathscr{C})=\beta\left(\mathscr{C}^{\prime}\right) \\
& \bar{x}_{i} \geq \min T \Rightarrow \min (\{i\} \cup T)=\min T \quad \Rightarrow \alpha(\mathscr{C})=\alpha\left(\mathscr{C}^{\prime}\right) \\
& \bar{x}_{i} \leq \min T \Rightarrow \min (\{i\} \cup T)=\bar{x}_{i} \quad \Rightarrow \bar{x}_{i} \leq \alpha\left(\mathscr{C}^{\prime}\right) \\
& \bar{x}_{i} \geq \max T \Rightarrow \max (\{i\} \cup T)=\bar{x}_{i} \quad \Rightarrow \beta\left(\mathscr{C}^{\prime}\right) \leq \bar{x}_{i} .
\end{aligned}
$$

Thus if $\min T \leq \bar{x}_{i} \leq \max T$, then $x(\mathscr{C})=x\left(\mathscr{C}^{\prime}\right)$ because $\alpha(\mathscr{C})=\alpha\left(\mathscr{C}^{\prime}\right)$ and $\beta(\mathscr{C})=\beta\left(\mathscr{C}^{\prime}\right)$. If $\bar{x}_{i} \leq \min T$ then $\alpha\left(\mathscr{C}^{\prime}\right) \leq \alpha(\mathscr{C})$ and $\beta(\mathscr{C})=\beta\left(\mathscr{C}^{\prime}\right)$ so $x\left(\mathscr{C}^{\prime}\right) \leq x(\mathscr{C})$ by Corollary 4 . Furthermore, $\bar{x}_{i} \leq \alpha\left(\mathscr{C}^{\prime}\right) \leq x\left(\mathscr{C}^{\prime}\right) \leq x(\mathscr{C})$. Finally, if $\max T \leq \bar{x}_{i}$, then $\alpha\left(\mathscr{C}^{\prime}\right)=\alpha(\mathscr{C})$ and $\beta(\mathscr{C}) \leq \beta\left(\mathscr{C}^{\prime}\right)$ so $x(\mathscr{C}) \leq x\left(\mathscr{C}^{\prime}\right)$ by Corollary 4. Furthermore $x(\mathscr{C}) \leq x\left(\mathscr{C}^{\prime}\right) \leq \beta\left(\mathscr{C}^{\prime}\right) \leq \bar{x}_{i}$. The result follows.

Turning to the comparative statics with respect to the recognition probabilities, we find that increasing the recognition probability of player $i$ at the expense of some other player cannot make player $i$ worse off. To see this, starting from the initial probability distribution $\mu$ consider increasing the recognition probability of player $i$ and decreasing that of player $j$, while keeping the rest of the distribution unchanged. Suppose without loss of generality that $\bar{x}_{i} \leq \bar{x}_{j}$. It is clear that the cumulative distribution function $\mu_{-}$does not change outside the interval $\left[\bar{x}_{i}, \bar{x}_{j}\right]$ and that it increases within the interval. Consequently, the characteristic function $\xi$ is unchanged outside the interval $\left[\bar{x}_{i}, \bar{x}_{j}\right]$ and it decreases within the interval. Therefore, if the limit of bargaining equilibria corresponding to the original recognition probabilities is outside the interval $\left[\bar{x}_{i}, \bar{x}_{j}\right]$, it is unchanged, while if it is within the interval, it shifts to the left, i.e. towards the ideal point of player $i$.

In the extreme case player $i$ is the only player entitled to make proposals $(\mu(i)=1)$. Under the unanimity acceptance rule the limit of bargaining equilibria will coincide with player $i$ 's ideal point irrespectively of preferences of the other players. 
We conclude this section with the observation that risk-aversion is disadvantageous in bargaining. To see this consider the differentiable utility functions $\bar{u}_{i}$ and $u_{i}$ satisfying Assumption (A1) and suppose $\bar{u}_{i}$ is more risk-averse than $u_{i}$. Then there is a concave non-negative differentiable function $f$ such that $\bar{u}_{i}(x)=f\left(u_{i}(x)\right)$ for each $x$. Then $\left|\bar{u}_{i}^{\prime}(x) / \bar{u}_{i}(x)\right| \leq\left|u_{i}^{\prime}(x) / u_{i}(x)\right|$ for each $x$. Using this fact it is easy to show that replacing the function $u_{i}$ by $\bar{u}_{i}$ results in a shift of the limit of bargaining equilibria away from the ideal point of player $i$.

\section{$6 \quad$ Extensions}

\subsection{Introducing the disagreement payoffs}

Implicitly assumed in the model of Section 2 is that the disagreement payoff, i.e. the payoff received by the players in the absence of agreement, is zero. Since we have assumed that the alternatives in $[0,1]$ give the individuals non-negative utility, everyone weakly prefers all the alternatives in $[0,1]$ to the disagreement payoff. As detailed below, the latter assumption can be weakened: it suffices to require that there be some alternative in $[0,1]$ which is strictly preferred by everyone to the disagreement payoff.

As in Banks and Duggan [1] and Cho and Duggan [5] we can introduce the disagreement payoffs explicitly into the model by redefining the payoff of the game $\Gamma(\delta)$ as follows: If the alternative $x$ is agreed upon in period $t$ define the payoff to player $i$ as $\left(1-\delta^{t}\right) d_{i}+\delta^{t} u_{i}(x)$, where the first term is the discounted sum of the disagreement payoffs $d_{i}$ over the periods $0, \ldots, t-1$, and the second term is the discounted sum of the payoffs $u_{i}(x)$ as of period $t$ (the sums are normalized by the factor of $1-\delta$ ). In the case of perpetual disagreement define the payoff to be $d_{i}$. It is not difficult to see that bargaining equilibria can be defined and analyzed as before with the function $u_{i}$ replaced everywhere by the function $v_{i}$ given by $v_{i}(x)=u_{i}(x)-d_{i}$.

To be consistent with the rest of the paper we continue to maintain the normalization $d_{i}=0$ for each $i \in N$. We replace Assumption (A1) by the following, weaker, assumption:

(A4) For each $i \in N$ the utility function $u_{i}:[0,1] \rightarrow \mathbb{R}$ is concave, continuous, and it attains its unique maximum at point $\bar{x}_{i}$. Moreover, there exists a point $p \in[0,1]$ such that $u_{i}(p)>0$ for each $i \in N$.

The version of Lemma 1 which applies under Assumption (A4) is the following.

Lemma 7 Assume (A2) and (A4). Let $\left(x_{\bullet}, A_{\bullet}\right)$ be a bargaining equilibrium of the game $\Gamma(\delta)$. The acceptance set $A_{C}$ of each coalition $C$ and the social acceptance set induced by the bargaining equilibrium are non-empty closed non-degenerate intervals.

One proves Lemma 7 by showing that each player's individual acceptance set contains the point $(1-\delta) p+\delta E\left(x_{\bullet}\right)$ whenever the point $p \in[0,1]$ is such that $u_{i}(p)>0$ for each $i \in N$. In Lemma 4 the upper bound on the length of $u_{i}(A)$ should be replaced by $(1-\delta) b / \nu$ 
where the constant $b$ has to be chosen to be larger than $u_{i}(x)$ for each $i \in N$ and each $x \in[0,1]$. Other results of Section 3 continue to be true exactly as they are stated.

When $\alpha<\beta$ we define the function $\varphi$ by Equations (4.4) and (4.5). For completeness, in the case $\alpha=\beta$ define $\varphi$ as $\varphi(x)=+\infty$ if $0 \leq x<\alpha, \varphi(\alpha)=0$, and $\varphi(x)=-\infty$ if $\alpha<x \leq 1$.

Lemma 8 The function $\varphi$ is a decreasing function on $[\alpha, \beta]$.

Proof. We first show that $\varphi_{-}$is a decreasing function. Let $\alpha<a<b<\beta$. Write $\ell_{i}(x)=-u_{i}(x) / l_{i}(x)$. We have for each coalition $C \in \mathscr{C}$

$$
\begin{aligned}
\max \left\{\ell_{i}(a) \mid i \in C, a<\bar{x}_{i}\right\} & \geq \max \left\{\ell_{i}(a) \mid i \in C, b<\bar{x}_{i}\right\} \\
& >\max \left\{\ell_{i}(b) \mid i \in C, b<\bar{x}_{i}\right\} \\
& \geq \varphi_{-}(b) .
\end{aligned}
$$

The first inequality of the chain is true since the set $\left\{i \in C: a<\bar{x}_{i}\right\}$ contains the set $\left\{i \in C: b<\bar{x}_{i}\right\}$. The second inequality is true because for each player $i$ with $b<\bar{x}_{i}$ we have $u_{i}(a)<u_{i}(b)$ and $l_{i}(a) \geq l_{i}(b)>0$ implying that $\ell_{i}(a)>\ell_{i}(b)$. The third inequality is true by the definition of $\varphi_{-}$. Since the above inequalities are true for each $C \in \mathscr{C}$ it follows that $\varphi_{-}(a)>\varphi_{-}(b)$. Similarly one proves that $\varphi_{+}$is a decreasing function.

To prove that $\varphi$ is a decreasing function we rewrite (4.5) as

$$
\varphi(x)=\sum \mu(i) \varphi_{i}(x), \text { where } \varphi_{i}(x)= \begin{cases}\varphi_{+}(x) & \text { if } x<\bar{x}_{i} \\ \varphi_{-}(x) & \text { if } \bar{x}_{i} \leq x\end{cases}
$$

Since $\varphi_{-}(x)<0<\varphi_{+}(x)$ for each $x$ it follows that $\varphi_{i}$ is a decreasing function for every $i$. It follows that $\varphi$ is a decreasing function.

The main results can then be restated as Theorems 6 below. The sketch of the proof of Theorem 6 is given in Appendix B. Theorem 6 implies that the limit of bargaining equilibria is a generalized zero of the function $\varphi$.

Theorem 6 Assume (A2) and (A4). Let $A=\left[x_{-}, x_{+}\right]$be the social acceptance set induced by a bargaining equilibrium of the game $\Gamma(\delta)$. Then $\varphi\left(x_{-}\right) \geq 0 \geq \varphi\left(x_{+}\right)$.

\subsection{Asymmetric discount factors}

Throughout the paper we have made the assumption that the players have a common discount factor. Consider instead a model where the player $i$ 's discount factor is given as a function $\delta_{i}=f_{i}(\delta)$ of the parameter $\delta$. The functions $f_{i}:[0,1] \rightarrow[0,1]$ are assumed to meet the following condition.

(A5) We have $0 \leq f_{i}(\delta)<1$ if $0 \leq \delta<1$ and $f_{i}(1)=1$. Furthermore, the function $f_{i}$ is continuously differentiable at the point 1 and $f_{i}^{\prime}(1)>0$. 
The version of Lemma 1 which applies in the model with asymmetric discount factors is the following.

Lemma 9 Assume (A1), (A2) and (A5). Let $\left(x_{\bullet}, A_{\bullet}\right)$ be a bargaining equilibrium of the game $\Gamma(\delta)$ inducing the social acceptance set $A$. Let $a=E\left(x_{\bullet}\right)$ and $\sigma=\max \left\{\delta_{i}\right\}$. Then $A$ is a closed interval containing the interval $[\sigma a, 1-\sigma+\sigma a]$.

Proof. Take any point $p$ in $[0,1]$. Using Jensen's inequality and the fact that $u_{i}(p) \geq 0$ we obtain the following chain of inequalities:

$$
u_{i}((1-\sigma) p+\sigma a) \geq(1-\sigma) u_{i}(p)+\sigma u_{i}(a) \geq \sigma \sum_{k \in N} \mu(k) u_{i}\left(x_{k}\right)=\sigma y_{i} \geq \delta_{i} y_{i}
$$

Hence the point $(1-\sigma) p+\sigma a$ is an element of the individual acceptance set of each player, and hence also of the social acceptance set. Taking $p=0$ and $p=1$ we see that both the point $\sigma a$ and $1-\sigma+\sigma a$ are contained in the social acceptance set. The result follows.

In Lemma 4 the upper bound on the length of $u_{i}(A)$ should be replaced by $\left(1-\delta_{i}\right) / \nu$. Other results of Section 3 continue to be true exactly as stated. Letting $\gamma_{i}=f_{i}^{\prime}(1)$ we define the characteristic function $\xi$ by Equations (4.1), (4.3) and the following equation which replaces (4.2):

$$
\xi_{-}(x)=\max _{C \in \mathscr{C}} \min _{i \in C}\left\{r_{i}(x) / \gamma_{i} u_{i}(x)\right\} \text { and } \xi_{+}(x)=\min _{C \in \mathscr{C}} \max _{i \in C}\left\{l_{i}(x) / \gamma_{i} u_{i}(x)\right\} .
$$

The main result is as follows.

Theorem 7 Assume (A1), (A2) and (A5). The limit of bargaining equilibria is unique and is equal to the (unique) generalized zero of the function $\xi$.

If the players' discount factors are different, it is no longer true that the generalized zero of the function $\xi$ is an element of the social acceptance set in each equilibrium of the game $\Gamma(\delta)$. In other words, the inequalities $\xi\left(x_{-}\right) \geq 0 \geq \xi\left(x_{+}\right)$are not generally true in our extended setting with asymmetric discount factors. Nevertheless, the proof of Theorem 7 follows the same strategy as the proof of Theorem 4: we establish a lower bound on $\xi\left(x_{-}\right)$ and an upper bound on $\xi\left(x_{+}\right)$and show that both bounds converge to zero as $\delta$ goes to one. The sketch of the proof of Theorem 7 is given in Appendix C.

One special case of (A5) warrants a special attention. Consider the functions $f_{i}$ given by the equation

$$
f_{i}(\delta)=\delta /\left(\gamma_{i}(1-\delta)+\delta\right), \text { where } \gamma_{i}>0 .
$$

In this case Lemmata 10-13 of Appendix A that make up the proof of Theorem 3 continue to be true exactly as they are stated for the modified characteristic function. Hence Theorem 3 also holds under Assumptions (A1), (A2) and (A5). Thus in the case of (6.2) our results carry over to the model with asymmetric discount factors almost effortlessly.

The two extensions we discussed in Subsections 6.1 and 6.2 cannot be easily combined. If players have different discount factors, and at the some of the alternatives give negative utilities, then the social acceptance set need not be an interval. 


\section{A The proof of Theorem 3}

Lemma 10 Let $\left(x_{\bullet}, A_{\bullet}\right)$ be a bargaining equilibrium of the game $\Gamma(\delta)$ inducing the social acceptance set $A=\left[x_{-}, x_{+}\right]$. If $0<x_{-}<1$, then

$$
\begin{aligned}
& -(1-\delta) \leq+\delta\left[x_{+}-E\left(x_{\bullet}\right)\right] \xi_{-}\left(x_{-}\right), \\
& +(1-\delta) \leq-\delta\left[x_{-}-E\left(x_{\bullet}\right)\right] \xi_{+}\left(x_{-}\right) .
\end{aligned}
$$

Proof. To prove the first inequality we have to show that there exists a coalition $C \in \mathscr{C}$ such that for each player $i$ in $C$ the inequality

$$
-(1-\delta) \leq \delta\left[x_{+}-E\left(x_{\bullet}\right)\right] r_{i}\left(x_{-}\right) / u_{i}\left(x_{-}\right)
$$

holds. Let $C_{+} \in \mathscr{C}$ be such that $x_{+} \in A_{C_{+}}$. We show that the inequality (A.3) holds for each player of the coalition $C_{+}$.

Let $i$ be a player of the coalition $C_{+}$. For each $k \in N$ we have the inequalities

$$
u_{i}\left(x_{+}\right)-u_{i}\left(x_{k}\right) \leq r_{i}\left(x_{k}\right)\left[x_{+}-x_{k}\right] \leq r_{i}\left(x_{-}\right)\left[x_{+}-x_{k}\right],
$$

where the first inequality is a defining inequality for the subgradient of the function $u_{i}$, and the second inequality follows from the fact that the right derivative is a non-increasing function, so that $r_{i}\left(x_{k}\right) \leq r_{i}\left(x_{-}\right)$for each $k \in N$, and the fact that $x_{k} \leq x_{+}$. (The right derivative at the point $x_{k}$ exists if $x_{k}<x_{+}$, because in that case $x_{k}$ is in ]0,1[. If $x_{k}=x_{+}$, then the extreme left and right hand sides of the above inequality are both zero.) Taking the expected value with respect to $k$ in both sides of the above inequality yields

$$
u_{i}\left(x_{+}\right)-y_{i} \leq r_{i}\left(x_{-}\right)\left[x_{+}-E\left(x_{\bullet}\right)\right] .
$$

The inequality (A.3) is trivially satisfied for any player $i$ with $x_{-}<\bar{x}_{i}$, because in this case $0<r_{i}\left(x_{-}\right)$. Assume that $\bar{x}_{i} \leq x_{-}$. Then the function $u_{i}$ is non-increasing on the interval $\left[x_{-}, x_{+}\right]$, in particular $u_{i}\left(x_{+}\right) \leq u_{i}\left(x_{-}\right)$. Since the coalition $C_{+}$unanimously approves of the alternative $x_{+}$, we have $\delta y_{i} \leq u_{i}\left(x_{+}\right)$for each $i \in C_{+}$. We have then the following inequalities:

$$
\begin{aligned}
0 & \leq u_{i}\left(x_{+}\right)-\delta y_{i}= \\
& =(1-\delta) u_{i}\left(x_{+}\right)+\delta\left[u_{i}\left(x_{+}\right)-y_{i}\right] \leq \\
& \leq(1-\delta) u_{i}\left(x_{+}\right)+\delta r_{i}\left(x_{-}\right)\left[x_{+}-E\left(x_{\bullet}\right)\right] \leq \\
& \leq(1-\delta) u_{i}\left(x_{-}\right)+\delta r_{i}\left(x_{-}\right)\left[x_{+}-E\left(x_{\bullet}\right)\right] .
\end{aligned}
$$

Rearranging yields (A.3).

To prove the second inequality we must show that for every coalition $C \in \mathscr{C}$ there exists a player $i$ in $C$ satisfying the inequality

$$
1-\delta \leq-\delta\left[x_{-}-E\left(x_{\bullet}\right)\right] l_{i}\left(x_{-}\right) / u_{i}\left(x_{-}\right) .
$$


Thus let $C$ be an arbitrary coalition from $\mathscr{C}$. First we argue that there exists a player $i \in C$ such that $u_{i}\left(x_{-}\right) \leq \delta y_{i}$. Suppose not. Then $\delta y_{i}<u_{i}\left(x_{-}\right)$for each $i \in C$. Since under the assumption of the lemma $x_{-}>0$ there exists a small enough $\epsilon>0$ such that $\delta y_{i}<u_{i}\left(x_{-}-\epsilon\right)$ for each $i \in C$. But then the point $x_{-}-\epsilon$ is an element of the social acceptance set $A$, contradicting the fact that $x_{-}$is the left endpoint of $A$.

Take any player $i \in C$ such that $u_{i}\left(x_{-}\right) \leq \delta y_{i}$. For each $k \in N$ we have the inequality

$$
u_{i}\left(x_{k}\right)-u_{i}\left(x_{-}\right) \leq l_{i}\left(x_{-}\right)\left[x_{k}-x_{-}\right]
$$

Taking the expected values on both sides with respect to $k$ yields

$$
y_{i}-u_{i}\left(x_{-}\right) \leq l_{i}\left(x_{-}\right)\left[E\left(x_{\bullet}\right)-x_{-}\right]
$$

Therefore,

$$
\begin{aligned}
0 & \geq u_{i}\left(x_{-}\right)-\delta y_{i}= \\
& =(1-\delta) u_{i}\left(x_{-}\right)+\delta\left[u_{i}\left(x_{-}\right)-y_{i}\right] \geq \\
& \geq(1-\delta) u_{i}\left(x_{-}\right)+\delta l_{i}\left(x_{-}\right)\left[x_{-}-E\left(x_{\bullet}\right)\right]
\end{aligned}
$$

Rearranging yields (A.4). This completes the proof.

Lemma 11 Let $\left(x_{\bullet}, A_{\bullet}\right)$ be a bargaining equilibrium of the game $\Gamma(\delta)$ inducing the social acceptance set $A=\left[x_{-}, x_{+}\right]$. Then the following inequalities hold:

$$
\begin{aligned}
& x_{+}-E\left(x_{\bullet}\right) \geq+\mu_{-}\left(x_{-}\right)\left[x_{+}-x_{-}\right] \\
& x_{-}-E\left(x_{\bullet}\right) \geq-\mu_{+}\left(x_{-}\right)\left[x_{+}-x_{-}\right] .
\end{aligned}
$$

Proof. Let $b_{i}$ be equal to $x_{-}$whenever $\bar{x}_{i} \leq x_{-}$and $x_{+}$otherwise. It is clear from Figure 1 that $x_{i} \leq b_{i}$ for each $i \in N$. It follows that

$$
E\left(x_{\bullet}\right) \leq E\left(b_{\bullet}\right)=\mu_{-}\left(x_{-}\right) x_{-}+\mu_{+}\left(x_{-}\right) x_{+} .
$$

Therefore,

$$
\begin{aligned}
& x_{+}-E\left(x_{\bullet}\right) \geq x_{+}-\left[\mu_{-}\left(x_{-}\right) x_{-}+\mu_{+}\left(x_{-}\right) x_{+}\right]=\mu_{-}\left(x_{-}\right)\left[x_{+}-x_{-}\right] \text {, } \\
& x_{-}-E\left(x_{\bullet}\right) \geq x_{-}-\left[\mu_{-}\left(x_{-}\right) x_{-}+\mu_{+}\left(x_{-}\right) x_{+}\right]=-\mu_{+}\left(x_{-}\right)\left[x_{+}-x_{-}\right] \text {. }
\end{aligned}
$$

The result follows.

We are now in a position to prove one of the inequalities of Theorem 3.

Lemma 12 Let $A=\left[x_{-}, x_{+}\right]$be the social acceptance set induced by a bargaining equilibrium of the game $\Gamma(\delta)$. Then $\xi\left(x_{-}\right) \geq 0$. 
Proof. Since the interval $A$ is non-degenerate by Lemma 1 , we know that $0 \leq x_{-}<1$. If $x_{-}=0$, there is nothing to prove because $\xi(0)=+\infty$ by the definition of $\xi$.

Suppose $0<x_{-}<1$. Inequality (A.2) of Lemma 10 implies that $0 \leq \xi_{+}\left(x_{-}\right)$. Therefore, if $0 \leq \xi_{-}\left(x_{-}\right)$, we immediately obtain the desired inequality $0 \leq \xi\left(x_{-}\right)$.

It remains to analyze the case where $x_{-}$is the interior point of $[0,1]$ and the inequalities $\xi_{-}\left(x_{-}\right) \leq 0 \leq \xi_{+}\left(x_{-}\right)$hold. We combine each of the two inequalities of Lemma 10 with those of Lemma 11, as follows:

$$
\begin{aligned}
& -(1-\delta) \leq \delta\left[x_{+}-E\left(x_{\bullet}\right)\right] \xi_{-}\left(x_{-}\right) \leq \delta \mu_{-}\left(x_{-}\right)\left[x_{+}-x_{-}\right] \xi_{-}\left(x_{-}\right) \\
& +(1-\delta) \leq-\delta\left[x_{-}-E\left(x_{\bullet}\right)\right] \xi_{+}\left(x_{-}\right) \leq \delta \mu_{+}\left(x_{-}\right)\left[x_{+}-x_{-}\right] \xi_{+}\left(x_{-}\right) .
\end{aligned}
$$

The first inequality in (A.7) is inequality (A.1) of Lemma 10. The second inequality in (A.7) is obtained using (A.5). The first inequality in (A.8) is inequality (A.2) of Lemma 10, and the second inequality in (A.8) is obtained using (A.6). Finally, adding up (A.7) and (A.8) and dividing by $\delta\left[x_{+}-x_{-}\right]$yields the result.

We complete the proof of Theorem 3 by establishing the second inequality of the theorem using a kind of a symmetry argument.

Lemma 13 Let $A=\left[x_{-}, x_{+}\right]$be the social acceptance set induced by a bargaining equilibrium of the game $\Gamma(\delta)$. Then $0 \geq \xi\left(x_{+}\right)$.

Proof. Given a point $x$ of $[0,1]$, let $\dot{x}=1-x$. Consider the game $\dot{\Gamma}(\delta)$ which has the same set $N$ of players, the same recognition probabilities $\mu$, the same set $\mathscr{C}$ of decisive coalitions as the game $\Gamma(\delta)$, and the utility function $\dot{u}_{i}(x)=u_{i}(\dot{x})$ for player $i$. Thus $\dot{\Gamma}(\delta)$ is essentially the same game as $\Gamma(\delta)$ with the alternatives permuted so that the alternative $x$ is mapped into $\dot{x}$. It is clear that the game $\dot{\Gamma}(\delta)$ has a bargaining equilibrium that induces the social acceptance set $\left[\dot{x}_{+}, \dot{x}_{-}\right]$. Let $\dot{\xi}$ denote the characteristic function of the game $\dot{\Gamma}(\delta)$. Since $\dot{x}_{+}$is a lower endpoint of the social acceptance set in a bargaining equilibrium of $\dot{\Gamma}(\delta)$, Lemma 12 applies to show that $\dot{\xi}\left(\dot{x}_{+}\right) \geq 0$. We complete the proof by showing that $\dot{\xi}(x) \leq-\xi(\dot{x})$ for each $x$. For then $\xi\left(x_{+}\right) \leq-\dot{\xi}\left(\dot{x}_{+}\right) \leq 0$, as desired.

If $x=0$ or $x=1$, the result follows from the definition of the characteristic function. So take an $x \in] 0,1\left[\right.$ and let $\dot{x}=1-x$. Let $\dot{l}_{i}, \dot{r}_{i}, \dot{\xi}_{-}, \dot{\xi}_{+}, \dot{\mu}_{-}$, and $\dot{\mu}_{+}$be defined as before for the game $\dot{\Gamma}(\delta)$. Then

$$
\begin{gathered}
\dot{l}_{i}(x)=-r_{i}(\dot{x}) \text { and } \dot{r}_{i}(x)=-l_{i}(\dot{x}), \\
\dot{\xi}_{-}(x)=-\xi_{+}(\dot{x}) \text { and } \dot{\xi}_{+}(x)=-\xi_{-}(\dot{x}) .
\end{gathered}
$$

Let $\mu_{o}(x)=\mu\left(\left\{i \in N \mid \bar{x}_{i}=x\right\}\right)$. Since the utility function $\dot{u}_{i}$ attains its maximum at the point $1-\bar{x}_{i}$, we have

$$
\begin{aligned}
& \dot{\mu}_{-}(x)=\mu\left(\left\{i \in N \mid 1-\bar{x}_{i} \leq x\right\}\right)=\mu_{+}(\dot{x})+\mu_{o}(\dot{x}), \\
& \dot{\mu}_{+}(x)=\mu\left(\left\{i \in N \mid x<1-\bar{x}_{i}\right\}\right)=\mu_{-}(\dot{x})-\mu_{o}(\dot{x}) .
\end{aligned}
$$


We can now express the characteristic function $\dot{\xi}$ as follows:

$$
\dot{\xi}(x)=-\xi(\dot{x})+\mu_{o}(\dot{x})\left[\xi_{-}(\dot{x})-\xi_{+}(\dot{x})\right] .
$$

By Lemma $5, \xi_{-}(\dot{x}) \leq \xi_{+}(\dot{x})$. We conclude that $\dot{\xi}(x) \leq-\xi(\dot{x})$, as desired.

\section{B The proof of Theorem 6}

If $\alpha=\beta$, then Theorem 6 is merely a restatement of Theorem 2. Hence throughout this section we assume $\alpha<\beta$.

Lemma 14 Let $\left(x_{\bullet}, A_{\bullet}\right)$ be a bargaining equilibrium of the game $\Gamma(\delta)$ inducing the social acceptance set $A=\left[x_{-}, x_{+}\right]$. If $\alpha<\beta$ then $x_{-}<\beta$ and $\alpha<x_{+}$.

Proof. We prove a stronger statement: If $\beta>0$ then $x_{-}<\beta$, and if $\alpha<1$ then $\alpha<x_{+}$. This clearly implies the result.

Take a player $i \in N$. For each $k \in N$ we have $\delta u_{i}\left(x_{k}\right) \leq \delta u_{i}\left(\bar{x}_{i}\right)<u_{i}\left(\bar{x}_{i}\right)$, where the first inequality holds since $\bar{x}_{i}$ is the maximum of the function $u_{i}$ on $[0,1]$, and the second inequality holds since $u_{i}\left(\bar{x}_{i}\right)>0$ by Assumption (A4). Taking the expectation with respect to $k$ we obtain $\delta y_{i}<u_{i}\left(\bar{x}_{i}\right)$.

It follows that if $\bar{x}_{i}>0$ then there exists an $\epsilon>0$ small enough such that $\delta y_{i}<u_{i}\left(\bar{x}_{i}-\epsilon\right)$, so that the point $\bar{x}_{i}-\epsilon$ is an element of the individual acceptance set of player $i$. Thus $x_{i}^{-}<\bar{x}_{i}$ whenever $\bar{x}_{i}>0$.

Now suppose that $\beta>0$. Then each decisive coalition $C \in \mathscr{C}$ has a player $i \in C$ with $\bar{x}_{i}>0$, in which case $x_{i}^{-}<\bar{x}_{i}$ as shown above. It follows that $\max \left\{x_{i}^{-}: i \in C\right\}<\max \left\{\bar{x}_{i}\right.$ : $i \in C\}$ for each $C \in \mathscr{C}$. Taking the minimum over $C \in \mathscr{C}$ on both sides of the inequality we obtain $x_{-}<\beta$. The proof that $\alpha<x_{+}$if $\alpha<1$ is similar.

Lemma 15 Let $\left(x_{\bullet}, A_{\bullet}\right)$ be a bargaining equilibrium of the game $\Gamma(\delta)$ inducing the social acceptance set $A=\left[x_{-}, x_{+}\right]$. If $\alpha<x_{-}<\beta$, then

$$
\begin{aligned}
& (1-\delta) \varphi_{+}\left(x_{-}\right) \geq \delta\left[x_{+}-E\left(x_{\bullet}\right)\right] . \\
& (1-\delta) \varphi_{-}\left(x_{-}\right) \geq \delta\left[x_{-}-E\left(x_{\bullet}\right)\right] .
\end{aligned}
$$

Proof. To prove the first inequality we show that there exists a coalition $C \in \mathscr{C}$ such that for each player $i \in C$ with $\bar{x}_{i}<x_{-}$for whom the inequality

$$
-(1-\delta) u_{i}\left(x_{-}\right) / r_{i}\left(x_{-}\right) \geq \delta\left[x_{+}-E\left(x_{\bullet}\right)\right]
$$

holds. This is done exactly as in the proof of Lemma 10. 
To prove the second inequality we must show that for every coalition $C \in \mathscr{C}$ there exists a player $i \in C$ with $x_{-}<\bar{x}_{i}$ satisfying the inequality

$$
-(1-\delta) u_{i}\left(x_{-}\right) / l_{i}\left(x_{-}\right) \geq \delta\left[x_{-}-E\left(x_{\bullet}\right)\right] .
$$

First one shows, exactly as in the proof of Lemma 10 that there exists a player $i \in C$ such that $u_{i}\left(x_{-}\right) \leq \delta y_{i}$. Take any player $i \in C$ such that $u_{i}\left(x_{-}\right) \leq \delta y_{i}$. We now argue that $x_{-}<\bar{x}_{i}$. For suppose on the contrary that $\bar{x}_{i} \leq x_{-}$. Then the function $u_{i}$ is decreasing on the interval $\left[x_{-}, x_{+}\right]$. Therefore for each point $x$ with $x_{-}<x \leq x_{+}$we have $u_{i}(x)<u_{i}\left(x_{-}\right)$ and so $u_{i}(x)<\delta y_{i}$. This shows that the individual acceptance set $A_{i}$ of player has at most one point, namely $x_{-}$, in common with the social acceptance set $A$. That is $A_{i} \cap A \subset\left\{x_{-}\right\}$. Consider now the acceptance set of the coalition $C$. By definition $A_{C}$ is a subset of both $A_{i}$ and $A$. Hence $A_{C} \subset\left\{x_{-}\right\}$, which contradicts the fact that $A_{C}$ is a non-empty nondegenerate interval (Lemma 7).

Take a player $i \in C$ with such that $u_{i}\left(x_{-}\right) \leq \delta y_{i}$. One shows, exactly as in the proof of Lemma 10, that player $i$ satisfies the inequality (B.4).

Lemma 16 Let $A=\left[x_{-}, x_{+}\right]$be the social acceptance set induced by a bargaining equilibrium of the game $\Gamma(\delta)$. Then $\varphi\left(x_{-}\right) \geq 0$.

Proof. As before we assume $\alpha<\beta$. Hence $x_{-}<\beta$ by Lemma 14. Moreover, if $x_{-} \leq \alpha$ then $\varphi\left(x_{-}\right)=+\infty$ by the definition of $\varphi$. It remains to consider the case $\alpha<x_{-}<\beta$. We combine each of the two inequalities of Lemma 15 with those of Lemma 11, as follows:

$$
\begin{aligned}
& (1-\delta) \varphi_{+}\left(x_{-}\right) \geq \delta\left[x_{+}-E\left(x_{\bullet}\right)\right] \geq+\delta \mu_{-}\left(x_{-}\right)\left[x_{+}-x_{-}\right] \\
& (1-\delta) \varphi_{-}\left(x_{-}\right) \geq \delta\left[x_{-}-E\left(x_{\bullet}\right)\right] \geq-\delta \mu_{+}\left(x_{-}\right)\left[x_{+}-x_{-}\right]
\end{aligned}
$$

Multiplying (B.5) by $\mu_{+}\left(x_{-}\right)$and (B.6) by $\mu_{-}\left(x_{-}\right)$and adding up yields $\varphi\left(x_{-}\right) \geq 0$.

The proof completes with Lemma 13 which needs no adjustments (except that $\xi$ is replaced everywhere by $\varphi$ ).

\section{C $\quad$ The proof of Theorem 7}

We prove Theorem 7 by establishing a lower bound on $\xi\left(x_{-}\right)$and an upper bound on $\xi\left(x_{+}\right)$, where $\left[x_{-}, x_{+}\right]$is the social acceptance set in a bargaining equilibrium of the game $\Gamma(\delta)$, and then showing that both bounds converge to zero as $\delta$ approaches one. To define the bounds we need some additional notation. Given $\delta$ such that $f_{i}(\delta)>0$ for each $i \in N$ let

$$
\begin{gathered}
g_{-}(\delta)=\min _{i \in N}\left\{\frac{1-f_{i}(\delta)}{f_{i}(\delta) \gamma_{i}}\right\} \text { and } g_{+}(\delta)=\max _{i \in N}\left\{\frac{1-f_{i}(\delta)}{f_{i}(\delta) \gamma_{i}}\right\} . \\
f(\delta)=\max _{i \in N} f_{i}(\delta) \\
e(\delta)=\left(g_{+}(\delta)-g_{-}(\delta)\right) /(1-f(\delta)) .
\end{gathered}
$$


Theorem 8 Suppose Assumptions (A2), (A4) and (A5) are satisfied. Suppose $f_{i}(\delta)>0$ for each $i \in N$. Let $A=\left[x_{-}, x_{+}\right]$be the social acceptance set induced by a bargaining equilibrium of the game $\Gamma(\delta)$. Then $e(\delta) \leq \xi\left(x_{-}\right)$and $\xi\left(x_{+}\right) \leq-e(\delta)$.

Theorem 7 follows from Theorem 8 by noticing that $e(\delta)$ converges to 0 as $\delta$ goes to 1. To see that this notice that both $g_{-}(\delta) /(1-\delta)$ and $g_{+}(\delta) /(1-\delta)$ converge to 1 while $[1-f(\delta)] /(1-\delta)$ converges to $\min \left\{f_{i}^{\prime}(1): i \in N\right\}$, which is positive by (A5). Below we sketch a proof of Theorem 8 .

Lemma 17 Suppose $f_{i}(\delta)>0$ for each $i \in N$. Let $\left(x_{\bullet}, A_{\bullet}\right)$ be a bargaining equilibrium of the game $\Gamma(\delta)$ inducing the social acceptance set $A=\left[x_{-}, x_{+}\right]$. If $0<x_{-}<1$, then

$$
\begin{aligned}
& -g_{+}(\delta) \leq+\left[x_{+}-E\left(x_{\bullet}\right)\right] \xi_{-}\left(x_{-}\right) \\
& +g_{-}(\delta) \leq-\left[x_{-}-E\left(x_{\bullet}\right)\right] \xi_{+}\left(x_{-}\right) .
\end{aligned}
$$

Proof. We first show, exactly as in the proof of Lemma 10, that there exists a coalition $C \in \mathscr{C}$ such that for each player $i$ in $C$ the inequality

$$
-\left(1-\delta_{i}\right) \leq \delta_{i}\left[x_{+}-E\left(x_{\bullet}\right)\right] r_{i}\left(x_{-}\right) / u_{i}\left(x_{-}\right)
$$

holds. Dividing by $\delta_{i} \gamma_{i}$ we obtain

$$
-g_{+}(\delta) \leq-\left(1-\delta_{i}\right) / \delta_{i} \gamma_{i} \leq\left[x_{+}-E\left(x_{\bullet}\right)\right] r_{i}\left(x_{-}\right) / \gamma_{i} u_{i}\left(x_{-}\right) .
$$

This establishes (C.1)

To prove the second inequality we show, exactly as in the proof of Lemma 10, that for every coalition $C \in \mathscr{C}$ there exists a player $i$ in $C$ satisfying the inequality

$$
1-\delta_{i} \leq-\delta_{i}\left[x_{-}-E\left(x_{\bullet}\right)\right] l_{i}\left(x_{-}\right) / u_{i}\left(x_{-}\right) .
$$

Dividing by $\delta_{i} \gamma_{i}$ we obtain

$$
g_{-}(\delta) \leq\left(1-\delta_{i}\right) / \delta_{i} \gamma_{i} \leq-\delta_{i}\left[x_{-}-E\left(x_{\bullet}\right)\right] l_{i}\left(x_{-}\right) / u_{i}\left(x_{-}\right) .
$$

This establishes (C.2)

Lemma 18 Let $A=\left[x_{-}, x_{+}\right]$be the social acceptance set induced by a bargaining equilibrium of the game $\Gamma(\delta)$. Then $\xi\left(x_{-}\right) \geq-e(\delta)$.

Proof. Since the interval $A$ is non-degenerate by Lemma 1 , we know that $0 \leq x_{-}<1$. If $x_{-}=0$, there is nothing to prove because $\xi(0)=+\infty$ by the definition of $\xi$.

Suppose $0<x_{-}<1$. Inequality (C.2) of Lemma 17 implies that $0 \leq \xi_{+}\left(x_{-}\right)$. Therefore, if $0 \leq \xi_{-}\left(x_{-}\right)$, we have $-e(\delta) \leq 0 \leq \xi\left(x_{-}\right)$. It remains to consider the case when $0<x_{-}<1$ 
and $\xi_{-}\left(x_{-}\right) \leq 0 \leq \xi_{+}\left(x_{-}\right)$. In this case we combine each of the two inequalities of Lemma 17 with those of Lemma 11, as follows:

$$
\begin{aligned}
& -g_{+}(\delta) \leq\left[x_{+}-E\left(x_{\bullet}\right)\right] \xi_{-}\left(x_{-}\right) \leq \mu_{-}\left(x_{-}\right)\left[x_{+}-x_{-}\right] \xi_{-}\left(x_{-}\right) \\
& +g_{-}(\delta) \leq-\left[x_{-}-E\left(x_{\bullet}\right)\right] \xi_{+}\left(x_{-}\right) \leq \mu_{+}\left(x_{-}\right)\left[x_{+}-x_{-}\right] \xi_{+}\left(x_{-}\right) .
\end{aligned}
$$

The first inequality in (C.3) is inequality (C.1) of Lemma 17. The second inequality in (C.3) is obtained using (A.5). The first inequality in (C.4) is inequality (C.2) of Lemma 17, and the second inequality in (C.4) is obtained using (A.6). Now we have the inequalities

$$
\xi\left(x_{-}\right) \geq-\frac{g_{+}(\delta)-g_{-}(\delta)}{x_{+}-x_{-}} \geq-\frac{g_{+}(\delta)-g_{-}(\delta)}{1-f(\delta)}=-e(\delta),
$$

where the first inequality is obtained by adding up (C.3) and (C.4) and dividing by $\left[x_{+}-x_{-}\right]$. To obtain the second inequality we notice that by Lemma 9 we have $1-f(\delta) \leq x_{+}-x_{-}$. The result follows.

The proof of Theorem 8 completes with Lemma 13, which requires no adjustments.

\section{References}

[1] Jeffrey S. Banks and John Duggan: A Bargaining Model of Collective Choice. American Political Science Review 94: 73-88, 2000.

[2] Ken Binmore, Ariel Rubinstein, and Asher Wolinsky: The Nash Bargaining Solution in Economic Modelling. The RAND Journal of Economics 17: 176-188, 1986.

[3] Volker Britz, P. Jean-Jacques Herings and Arkadi Predtetchinski: Non-Cooperative Support for the Asymmetric Nash Bargaining Solution. Journal of Economic Theory, doi:10.1016/j.jet.2010.04.003.

[4] Daniel Cardona and Clara Ponsatí: Bargaining One-Dimensional Social Choices. Journal of Economic Theory 137, 627-651, 2007.

[5] Seok-Ju Cho and John Duggan: Uniqueness of Stationary Equilibria in a OneDimensional Model of Bargaining. Journal of Economic Theory 113: 118-130, 2003.

[6] Olivier Compte And Philippe Jehiel: Bargaining over Randomly Generated Offers: A new perspective on multi-party bargaining. Levine's Bibliography 122247000000000739, UCLA Department of Economics, 2004.

[7] Hülya ERaslan: Uniqueness of Stationary Equilibrium Payoffs in the BaronFerejohn Model. Journal of Economic Theory 103: 11-30, 2002.

[8] Hülya Eraslan and Antonio Merlo: Majority Rule in a Stochastic Model of Bargaining. Journal of Economic Theory 103: 31-48, 2002. 
[9] Haruo Imai and Hannu Salonen: The Representative Nash Solution for Twosided Bargaining Problems. Mathematical Social Sciences 39: 349-365, 2000.

[10] Sergiu Hart and Andreu Mas-Colell: Bargaining and Value. Econometrica 64: 357-380, 1996.

[11] P. Jean-Jacques Herings and Arkadi Predtetchinski: One-dimensional Bargaining with Markov Recognition Probabilities. Journal of Economic Theory 145: 189-215, 2010.

[12] Tasos KalandRAKIS: Regularity of pure strategy equilibrium points in a class of bargaining games. Economic Theory 28: 309-329, 2006.

[13] Klaus Kultti and Hannu Vartiainen: Von Neumann-Morgenstern stable sets, discounting, and Nash bargaining. Journal of Economic Theory 137: 721-728, 2007.

[14] Annick Laruelle and Federico Valenciano: Noncooperative foundations of bargaining power in committees and the Shapley-Shubik index. Games and Economic Behaviour 65, 242-255, 2009.

[15] Antonio Merlo and Charles Wilson: A Stochastic Model of Sequential Bargaining with Complete Information. Econometrica 63: 371-399, 1995.

[16] Antonio Merlo and Charles Wilson: Effcient delays in a stochastic model of bargaining. Economic Theory 11: 39-55, 1998.

[17] Toshiji Miyakawa: Non-cooperative Foundation of $n$-Person Asymmetric Nash Bargaining Solution. Osaka University of Economics, working paper No. 2006-2. 